\title{
PROPERTIES OF GROWTH FOR SOLUTIONS OF DIFFERENTIAL EQUATIONS OF DYNAMICAL TYPE
}

\author{
BX \\ W. J. TRJITZINSKY
}

1. Introduction. In this work we shall investigate properties of "growth" of solutions of differential systems, with particular reference to systems of the type of importance in dynamics. The systems of the latter kind are nonlinear. A considerable part of the developments for the nonlinear problem can be based on the theory of the problem obtained by retaining the linear part, only, of the nonlinear system in question. In $\$ \S 3$ and 4 we shall give developments for linear systems. This treatment is followed by consideration of nonlinear systems of a very general type, given in $\$ \S 5,6$. It is not our main purpose, at this time, to investigate problems of dynamical stability and instability. However, it is to be noted that Theorem 6.1 presents an extended case of conditional stability.

Of importance from our present point of view are a number of contributions, of which we shall mention certain memoirs due to A. Liapounoff $\left(^{1}\right)$, P. Bohl $\left({ }^{2}\right)$, E. Cotton $\left({ }^{3}\right)$, O. Perron $\left({ }^{4}\right)$.

In $\$ 2$ we investigate growth of functions on the basis of "characteristic numbers," defined with respect to any "admissible" function (cf. Definitions $2.1,2.3)$. The developments for linear differential systems are given with the aid of characteristic numbers ( $\$ 3)$ and "product integration" $(\$ 4)$. In the study of nonlinear systems use is made of characteristic numbers $(\$ 5)$ and of the method of successive approximations (\$6).

Presented to the Society, September 11, 1940; received by the editors April 1, 1940, and, in revised form, September 25, 1940.

(1) A. Liapounoff, Problème général de la stabilité du mouvement (translated from Russian by E. Davaux), Annales de la Faculté des Sciences de Toulouse (2), vol. 9 (1907), pp. 203-474, originally published by the Société Mathématique de Kharkow in 1892. This work will be referred to as (L).

(2) P. Bohl, Sur certaines équations différentielles d'un type gênéral utilisable en mécanique (translated from Russian by Mlle Tarnarider), Bulletin de la Société Mathématique de France, vol. 38 (1910), pp. 5-138; also Ueber die Bewegung eines mechanischen Systems in der Nähe einer Gleichgewichtslage, Journal für die reine und angewandte Mathematik, vol. 127 (1904), pp. 179-276.

(3) E. Cotton, Sur les solutions asymptotiques des équations différentielles, Annales Scientifique de l'École Normale Supérieure, vol. 28 (1911), pp. 473-521.

(4) O. Perron, Ueber Stabilität und asymptotisches Verhalten der Integrale von Differentialgleichungssystemen, Mathematische Zeitschrift, vol. 29 (1928), pp. 129-160; also Die Stabilitätsfrage bei Differentialgleichungen, ibid., vol. 32 (1930), pp. 703-728. 
The main results are embodied in Theorems $2.1,2.2,3.1,3.2,4.1,4.2,4.3$, $5.1,5.2,6.1$. There is an important application given subsequent to Theorem 6.1.

2. Growth of functions. In the sequel, unless the contrary is stated, all the functions and numbers will be supposed to be real, the functions being defined on the interval $\left(t_{0},+\infty\right)$.

It will be convenient to introduce the following definitions.

Definition 2.1. A function $\psi(t)$, continuous for $t \geqq t_{0}$, monotone non-decreasing for $t \geqq t_{0}$, and such that

$$
\lim \psi(t)=+\infty \quad(\text { for } t \rightarrow+\infty)
$$

will be said to be "admissible."

Definition 2.2. A function $\gamma(t)$ will be said to be identically zero, provided there exists a number $t_{1}\left(\geqq t_{0}\right)$ so that $\gamma(t)=0$ for all $t \geqq t_{1}$.

Definition 2.3. A function $x(t)$ will be said to have a "characteristic number" $\lambda$, with respect to an admissible function $\psi(t)$, provided

$$
\lim \sup \left|x(t) \psi^{\lambda+\epsilon}(t)\right|=\infty, \quad \lim \left|x(t) \psi^{\lambda-\epsilon}(t)\right|=0
$$

$($ as $t \rightarrow+\infty)$ for all $\epsilon>0$. We shall employ the following notation for this number:

$$
\lambda=C(x(t) \mid \psi(t)) .
$$

When in the first relation (2.1) one may replace lim sup by lim, it will be said that $\lambda$ is a characteristic number in the strong sense; this number will then be designated by

$$
\lambda=C_{s}(x(t) \mid \psi(t)) .
$$

It is to be noted that characteristic numbers in the sense of (2.2), with $\psi(t)=\exp t$, have been previously used by Liapounoff (cf. (L)).

Definition 2.4. Given a function $f(t)$, form the limits

$$
U\left(t_{1}\right)=\lim \sup |f(t)|, \quad u\left(t_{1}\right)=\lim \inf |f(t)| \quad\left(\text { for } t \geqq t_{1}\right),
$$

where $t_{1}$ is any number $\geqq t_{0}$. We shall write

$$
\left.\bar{L}|f(t)|=\lim U\left(t_{1}\right), \quad \underline{L}|f(t)|=\lim u\left(t_{1}\right) \quad \text { (as } t_{1} \rightarrow+\infty\right) .
$$

Clearly, $U\left(t_{1}\right)$ is monotone non-increasing and $u\left(t_{1}\right)$ is monotone non-decreasing.

The advantage in the use of $\bar{L}, \underline{L}$ in place of lim sup, lim inf lies in the fact that the first two symbols are more descriptive of the behavior of $f(t)$ in the neighborhood of $t=+\infty$.

We shall now prove the following theorem. 
Theorem 2.1. Let $x(t)$ be continuous for $t \geqq t_{0}$ and be not identically zero. Then $x(t)$ has a finite characteristic number with respect to some admissible function $\psi(t)$.

Suppose first the $|x(t)|$ is not uniformly bounded. One then has

$$
\lim \sup |x(t)|=\bar{L}|x(t)|=\infty \text {. }
$$

There exists a non-empty class $K$ of functions $q(t)$, continuous, monotone non-decreasing and such that

$$
|x(t)| \leqq q(t) .
$$

In the class $K$ there is a "least" function $\psi(t)$; that is, for every $t\left(\geqq t_{0}\right)$ the value $\psi(t)$ is the least value which the functions of $K$ may assume. Clearly, in view of $(2.3) \psi(t)$ is admissible and

$$
|x(t)| \leqq \psi(t)
$$

moreover, there exists a set of values

$$
t_{0}<t_{1}<\cdots<t_{\nu}<\cdots,
$$

with $\lim _{\nu} t_{\nu}=\infty$, so that

$$
\psi\left(t_{v}\right)=\left|x\left(t_{v}\right)\right| \quad(\nu=1,2, \cdots) .
$$

One has

$$
l_{\epsilon}(t)=\left|x(t) \psi^{-1+\epsilon}(t)\right|=\left|x(t) \psi^{-1}(t)\right| \psi^{\epsilon}(t) ;
$$

hence

$$
\begin{array}{lrl}
l_{\epsilon}\left(t_{\nu}\right)=\psi^{\epsilon}\left(t_{\nu}\right) & \rightarrow \infty & (\text { all } \epsilon>0 ; \text { as } \nu \rightarrow \infty), \\
\lim \sup l_{\epsilon}(t)=\infty & (\text { all } \epsilon>0) .
\end{array}
$$

On the other hand, by (2.4)

$$
l_{-\dot{\epsilon}}(t)=\left|x(t) \psi^{-1-\epsilon}(t)\right| \leqq \psi^{-\epsilon}(t) .
$$

Whence

$$
\lim l_{-\epsilon}(t)=0
$$

Thus, when $|x(t)|$ is unbounded, one has

$$
C(x(t) \mid \psi(t))=-1,
$$

where $\psi(t)$ is the function referred to in (2.4).

The alternative to the hypothesis implied in (2.3) is

$$
|x(t)| \leqq \beta,
$$

(finite $\beta>0$ ). 
One then may have, when $t_{1}\left(\geqq t_{0}\right)$ is taken sufficiently great,

$$
0<\alpha \leqq|x(t)| \leqq \beta \quad\left(t \geqq t_{1} ; \text { constant } \alpha\right) ;
$$

then, on letting $\psi(t)$ denote any admissible function, it is inferred that

$$
\left|x(t) \psi^{\epsilon}(t)\right| \geqq \alpha \psi^{\epsilon}(t), \quad\left|x(t) \psi^{-\epsilon}(t)\right| \leqq \beta \psi^{-\epsilon}(t),
$$

which implies

$$
\lim \left|x(t) \psi^{\epsilon}(t)\right|=\infty, \quad \lim \left|x(t) \psi^{-\epsilon}(t)\right|=0
$$

for all $\epsilon>0$. Whence, under (2.6),

$$
C_{s}(x(t) \mid \psi(t))=0
$$

for all admissible functions $\psi(t)$.

The remaining subcase of $(2.5)$ is

$$
|x(t)| \leqq \beta, \quad \underline{L}|x(t)|=0 .
$$

The latter relation will imply existence of values

$$
\left(t_{0}<\right) t_{1}^{\prime}<t_{2}^{\prime}<\cdots<t_{\nu}^{\prime}<\cdots ; \lim _{\nu} t_{\nu}^{\prime}=\infty
$$

for which

$$
\lim _{\nu}\left|x\left(t_{\nu}^{\prime}\right)\right|=0
$$

This follows from the fact that the equality $\underline{L}|x(t)|=0$ signifies that the function

$$
u(T)=\lim \inf |x(t)| \quad(t \geqq T)
$$

is zero for all $T \geqq t_{0}$. In consequence of the continuity of $x(t)$ and inasmuch as $x(t)$ is not identically zero one may choose the $t_{v}^{\prime}$ so that

$$
\left|x\left(t_{\nu}^{\prime}\right)\right|>0 \quad(\nu=1,2, \cdots) .
$$

Amongst the $\left(t_{\nu}^{\prime}\right)$ one may select an infinite subsequence $\left(t_{\nu}\right)(\nu=1,2, \cdots)$ so that

$$
\left|x\left(t_{1}\right)\right| \geqq\left|x\left(t_{2}\right)\right| \geqq \cdots \geqq\left|x\left(t_{v}\right)\right| \geqq \cdots ;
$$

necessarily

$$
\left|x\left(t_{v}\right)\right|>0, \quad \lim _{v}\left|x\left(t_{v}\right)\right|=0 .
$$

Let $h(u)$ be any admissible function, defined for

and such that

$$
u \geqq\left|x\left(t_{1}\right)\right|^{-1}=u_{0}
$$

$$
\left.\lim _{\nu}\left|x\left(t_{\nu}\right) h^{\epsilon}\left(\left|x\left(t_{\nu}\right)\right|^{-1}\right)\right|=\infty \quad \text { (all } \epsilon>0\right)
$$


For example, $h(u)=\exp u$ is such a function. In consequence of (2.7)

$$
\gamma_{\nu}=h\left(\left|x\left(t_{v}\right)\right|^{-1}\right) \leqq \gamma_{v+1} \quad(\nu=1,2, \cdots) ;
$$

moreover, by (2.7a) $\lim \gamma_{\nu}=\infty$. Hence it is possible to construct an admissible function $\psi(t)$ for which

$$
\psi\left(t_{v}\right)=\gamma: \quad(\nu=1,2, \cdots) .
$$

On writing

$$
\lambda_{\epsilon}(t)=\left|x(t) \psi^{\epsilon}(t)\right|
$$

it is observed that

$$
\lambda_{\epsilon}\left(t_{v}\right)=\mid x\left(t_{v}\right) h^{\epsilon}\left(\left|x\left(t_{v}\right)\right|^{-1}\right) .
$$

Whence in consequence of (2.8)

$$
\lim _{\nu} \lambda_{\epsilon}\left(t_{\nu}\right)=\infty
$$

Together with (2.9), this implies that

$$
\lim \sup \left|x(t) \psi^{\epsilon}(t)\right|=\infty
$$

for the admissible function $\psi(t)$ introduced above.

On the other hand, on noting the relations

$$
|x(t)| \leqq \beta, \quad \underline{L}|x(t)|=0,
$$

it is observed that the function of (2.9) satisfies

$$
\lambda_{-\epsilon}(t) \leqq \beta \psi^{-\epsilon}(t) ;
$$

thus

$$
\lim \lambda_{-\epsilon}(t)=0
$$

Hence, for the subcase under consideration, we have

$$
C(x(t) \mid \psi(t))=0,
$$

where $\psi(t)$ is an admissible function with properties as described above.

This establishes Theorem 2.1.

THEOREM 2.2. Let $x(t)$ be continuous and be not identically zero. In order that

$$
C(x(t) \mid \psi(t))=0
$$

for all admissible functions $\psi(t)$ it is necessary and sufficient that

$$
\bar{L}|x(t)| \neq 0, \quad \neq \infty \text {. }
$$

To prove that (2.10) implies (2.11) suppose, if possible, that

$$
\bar{L}|x(t)|=\infty \text {. }
$$


There exists then an admissible function $q(t)(>0)$ such that

$$
|x(t)| \leqq q(t), \quad q\left(t_{v}\right)=\left|x\left(t_{v}\right)\right| \rightarrow \infty \quad(\text { as } \nu \rightarrow \infty),
$$

where the $t_{v}$ are certain numbers satisfying

$$
t_{0}<t_{1}<\cdots ; \quad \lim t_{\nu}=\infty .
$$

Let $\phi(t)$ be any admissible function for which

$$
\lim q(t) \psi^{-\epsilon}(t)=\infty \quad(\text { all } \epsilon>0 \text {; as } t \rightarrow \infty) .
$$

One may take $\phi(t)=q(t)$ and $0<\epsilon<1$. We write

$$
\lambda_{-\epsilon}(t)=\left|x(t) \phi^{-\epsilon}(t)\right|=\left|\frac{x(t)}{q(t)}\right| q(t) \phi^{-\epsilon}(t)
$$

and note that in view of the preceding

Whence

$$
\left|\frac{x\left(t_{v}\right)}{q\left(t_{v}\right)}\right|=1 \text {. }
$$

$$
\lambda_{-\epsilon}\left(t_{v}\right)=q\left(t_{v}\right) \phi^{-\epsilon}\left(t_{v}\right) \rightarrow \infty \quad(\text { as } \nu \rightarrow \infty)
$$

for all $\epsilon>0$ and

$$
\lim \sup \lambda_{-\epsilon}(t)=\infty
$$

for any function $\phi(t)$ of the above description. The latter relation is contrary to the hypothesis that (2.10) holds for all admissible functions $\psi(t)$. Thus (2.12) is impossible and we have

$$
|x(t)| \leqq \beta<\infty .
$$

Still assuming (2.10) it is now observed that failure of (2.11) is conceivable only when (2.13) holds, while

$$
\bar{L}|x(t)|=0 .
$$

Now the latter relation implies that $\lim x(t)=0$ (as $t \rightarrow \infty)$. Amongst the continuous monotone non-increasing functions $c(t)$, such that

$$
|x(t)| \leqq c(t)
$$

there is a least function $h(t)$ with the same properties. We have

$$
h(t)=\lim \sup |x(\tau)| \quad(\tau \geqq t) .
$$

Since

$$
|x(t)| \leqq h(t)
$$


and since $x(t)$ is not identically zero, it is deduced that $h(t)>0$; moreover,

$$
\lim _{t \rightarrow \infty} h(t)=0 \text {. }
$$

It is observed that for some admissible $\phi(t)$ we have

$$
\lambda_{\epsilon}(t)=\left|x(t) \phi^{\epsilon}(t)\right| \rightarrow 0 \quad(\text { all } \epsilon>0 ; \text { as } t \rightarrow \infty) .
$$

In fact, one may take

$$
\phi(t)=-\log h(t) .
$$

Whence (2.10) is impossible for some admissible functions and (2.13a) cannot hold. Accordingly, (2.10) implies (2.11).

To establish the converse we assume (2.11) and proceed to deduce (2.10).

The second relation (2.11) implies that

$$
|x(t)| \leqq \beta<\infty .
$$

Hence, with $\psi(t)$ denoting any admissible function, one has

$$
\lambda_{-\epsilon}(t)=\left|x(t) \psi^{-\epsilon}(t)\right| \leqq \beta \psi^{-\epsilon}(t)
$$

and

$$
\lim \lambda_{-\epsilon}(t)=0 \quad(\text { all } \epsilon>0) .
$$

On the other hand, on writing

$$
\bar{L}|x(t)|=\alpha(>0)
$$

and on letting $\alpha_{0}$ denote any constant such that $0<\alpha_{0}<\alpha$, it is deduced that there exist values

$$
t_{1}<t_{2}<\cdots<t_{\nu}<\cdots \quad\left(t_{0}<t_{1}\right)
$$

such that

$$
\lim _{\nu} t_{\nu}=+\infty, \quad\left|x\left(t_{\nu}\right)\right| \geqq \alpha_{0} \quad(\nu=1,2, \cdots) .
$$

This follows from the continuity of $x(t)$. Let $\psi(t)$ again be any admissible function. By the above relations

$$
\lambda_{\epsilon}\left(t_{v}\right) \geqq \alpha_{0} \psi^{\epsilon}\left(t_{v}\right) .
$$

The last member, here, tends to $+\infty$ with $t$, for all $\epsilon>0$. Thus

$$
\limsup _{t \rightarrow \infty} \lambda_{\epsilon}(t)=\infty
$$

Together with a previous relation for $\lambda_{-\epsilon}(t)$ this establishes $(2.10)$, which completes the proof of Theorem 2.2.

We shall define the characteristic number (with respect to an admissible 
function $\psi(t)$ of a finite set of functions $x_{j}(t),(j=1, \cdots, m)$ as the least one of the characteristic numbers of the $x_{j}(t)$. This is in agreement with Liapounoff's definition for the case of $\psi(t)=\exp t$. Our designation for the characteristic number (with respect to $\psi(t)$ of functions $x_{1}(t), \cdots, x_{m}(t)$ will be

$$
C\left(x_{1}, \cdots, x_{m}(t) \mid \psi(t)\right) .
$$

3. Characteristic numbers for linear systems. Consider the linear differential system

$$
\frac{d x_{i}}{d t}=p_{1, i}(t) x_{1}+\cdots+p_{n, i}(t) x_{n} \quad(i=1, \cdots, n)
$$

where the $p_{i, j}(t)$ are continuous for $t \geqq t_{0}$.

Let $p(t)$ be the "least" function such that

$$
\left|p_{i, j}(t)\right| \leqq p(t) \quad(i, j=1, \cdots, n) .
$$

Necessarily $p(t)$ is continuous. Inasmuch as $p(t) \geqq 0$, the function

$$
\phi(t)=\int_{t_{0}}^{t} p(t) d t
$$

will be continuous, monotone non-decreasing.

The transformation

$$
\tau=\phi(t)
$$

applied to (3.1), will yield

where

$$
\frac{d x_{i}}{d \tau}=h_{1, i}(\tau) x_{1}+\cdots+h_{n, i}(\tau) x_{n} \quad(i=1, \cdots, n)
$$

$$
h_{i, j}(\tau)=\frac{p_{i, j}(t)}{p(t)} .
$$

In view of (3.3) and (3.2a) to the interval $\left(t_{0} \leqq t<\infty\right)$ corresponds the interval $\left(0 \leqq \tau \leqq \tau^{\prime}\right)$, with

$$
\tau^{\prime}=\int_{t_{0}}^{\infty} p(t) d t,
$$

the convention being made that when the latter integral diverges one has $\tau^{\prime}=\infty$. In any case, by virtue of (3.2),

$$
\left|h_{i, j}(\tau)\right| \leqq 1 \quad\left(0 \leqq \tau \leqq \tau^{\prime}\right) .
$$

Lemma 3.1. Every linear differential system (3.1) is transformed by (3.3) [(3.2a), (3.2)] into a system of the same type with coefficients uniformly bounded 
in absolute value on an interval which is infinite or finite, according as the integral

$$
\int_{t_{0}}^{\infty} p(t) d t
$$

diverges or converges.

We recall now a certain theorem, due to Liapounoff( $\left.{ }^{5}\right)$. In the notation at the end of $\$ 2$, the theorem states that for every solution

$$
x_{1}(t), \cdots, x_{n}(t)
$$

of a system (3.1), whose coefficients are continuous and uniformly bounded in absolute value for $t \geqq t_{0}$, the number

$$
C\left(x_{1}(t), \cdots, x_{n}(t) \mid e^{t}\right)
$$

is finite.

This fact, together with Lemma 3.1, enables us at once to make the following inference with respect to characteristic numbers of solutions of any system (3.1).

We form the function $p(t)$, as stated in connection with (3.2). If the integral (3.4) converges, then $p(t)$ is uniformly bounded for $t \geqq t_{0}$; the same will be true of the $\left|p_{i, j}(t)\right|(i, j=1, \cdots, n)$ and, consequently, for every solution, $x_{1}(t), \cdots, x_{n}(t)$, of (3.1) the characteristic number (3.5) will be finite. If, on the other hand, the integral (3.4) diverges, one may assert that for every solution $x_{1}(t), \cdots, x_{n}(t)$ of (3.1) the characteristic number

$$
C\left(x_{1}(t), \cdots, x_{n}(t) \mid \exp \left\{\int_{t_{0}}^{t} p(t) d t\right\}\right)
$$

will be finite.

We shall give greater precision to the above results by examining more closely the lines of approach used in (L), p. $229 \mathrm{ff}$.

We shall need the following lemma, which will be stated without proof.

Lemma 3.2. Let $\psi(t)$ be an admissible function. Then

$$
C\left(x_{1}^{2}+\cdots+x_{n}^{2} \mid \psi(t)\right)=2 C\left(x_{1}, \cdots, x_{n} \mid \psi(t)\right),
$$

provided at least one of the above characteristic numbers is finite.

In this connection we note that, given any set of functions $x_{j}(t)$ $(j=1, \cdots, n)$, continuous and not all identically zero, there exist admissible functions $\psi(t)$ for which $C\left(x_{1}^{2}+\cdots+x_{n}^{2} \mid \psi(t)\right)$ is finite. This follows from Theorem 2.1.

(b) (L), p. 229. 
In view of Lemma 3.1 there is no loss of generality in considering the system (3.1) with coefficients subject to inequalities

$$
\left|p_{i, j}(t)\right| \leqq b<\infty .
$$

By Liapounoff's result ((L), p. $229 \mathrm{ff}$.), we merely know that every solution $x_{j}=x_{j}(t),(j=1, \cdots, n)$, of $(3.1)$ has a finite characteristic number with respect to exp $t$, provided (3.7) holds. We shall obtain bounds, depending on $b$, for the characteristic numbers of solutions.

Let $x_{1}, \cdots, x_{n}$ be a solution of (3.1). With Liapounoff, we employ the transformation

$$
z_{i}=x_{i} e^{\lambda t} \quad(\lambda \text { an indeterminate number })
$$

which yields

$$
\frac{d z_{i}}{d t}=p_{1, i}(t) z_{1}+\cdots+\left(p_{i, i}(t)+\lambda\right) z_{i}+\cdots+p_{n, i}(t) z_{n} \quad(i=1, \cdots, n)
$$

and, on writing $u=z_{1}^{2}+\cdots+z_{n}^{2}$, we obtain

$$
\frac{1}{2} \frac{d u}{d t}=A(\lambda)+B(\lambda)=\Gamma(\lambda)
$$

where

(3.9a) $\quad A(\lambda)=\sum_{i}\left(p_{i, i}(t)+\lambda\right) z_{i}^{2}, \quad B(\lambda)=\sum_{s \neq \sigma}\left(p_{s, \sigma}(t)+p_{\sigma, s}(t)\right) z_{8} z_{\sigma}$.

Using the inequality of Schwartz, it can be proved that

$$
\sum_{s \neq \sigma}\left|\alpha_{s} \alpha_{\sigma}\right| \leqq(n-1)\left(\alpha_{1}^{2}+\cdots+\alpha_{n}^{2}\right) \quad(s, \sigma=1, \cdots, n)
$$

for any numbers $\alpha_{1}, \cdots, \alpha_{n}$. Hence by (3.9a) and (3.7)

$$
|B(\lambda)| \leqq 2 b \sum_{s \neq \sigma}\left|z_{s} z_{\sigma}\right| \leqq 2(n-1) b u \text {. }
$$

Define $\lambda_{0}$ as

$$
\lambda_{0}=(2 n-1) b+\eta / 2
$$

$(\eta>0)$

In consequence of (3.9a) and (3.7)

$$
\left.p_{i, i}(t)+\lambda \geqq \lambda_{0}-b>0 \quad \text { (for } \lambda \geqq \lambda_{0}\right)
$$

and

$$
\left.A(\lambda) \geqq\left(\lambda_{0}-b\right) u>0 \quad \text { (for } \lambda \geqq \lambda_{0}\right) .
$$

By virtue of (3.10) and of the above, from (3.9) we deduce 


$$
\Gamma(\lambda) \geqq\left[\left(\lambda_{0}-b\right)-2(n-1) b\right] u=\frac{\eta}{2} u
$$

for all $\lambda \geqq \lambda_{0}$ and for all $t \geqq t_{0}$. Whence by (3.9)

$$
u \geqq c e^{\eta t} \quad\left(\lambda \geqq \lambda_{0} ; \text { constant } c>0\right) .
$$

It is similarly established that

$$
\Gamma(\lambda) \leqq-\frac{\eta}{2} u \quad\left(\text { for all } \lambda \leqq-\lambda_{0}\right),
$$

which yields

$$
u \leqq c_{1} e^{-\eta t} \quad\left(\lambda \leqq-\lambda_{0} ; \text { constant } c_{1}>0\right) .
$$

By (3.8)

$$
u=e^{2 \lambda t} v, \quad v=x_{1}^{2}+\cdots+x_{n}^{2} ;
$$

here the $x_{j}(j=1, \cdots, n)$ are independent of $\lambda$ and constitute a solution of (3.1). Thus, in view of (3.12) and (3.12a),

$$
\lim e^{2 \lambda t} v=+\infty \quad\left(\text { all } \lambda \geqq \lambda_{0}\right), \quad \lim e^{2 \lambda t} v=0 \quad\left(\text { all } \lambda \leqq-\lambda_{0}\right),
$$

where $\lambda_{0}$ is from (3.11). Inasmuch as $\eta(>0)$ may be taken arbitrarily small, the above relations can be stated in the form

(3.13) $\lim e^{2 \lambda t} v=+\infty($ all $\lambda>(2 n-1) b), \lim e^{2 \lambda t} v=0(\operatorname{all} \lambda<-(2 n-1) b)$.

An inequality

$$
\mu=C\left(v \mid e^{t}\right)>2(2 n-1) b
$$

is impossible. In fact, suppose (3.14) holds. It is noted that

$$
e^{(\mu-\epsilon) t} v \doteq\left|e^{2[(2 n-1) b+\epsilon] t} v\right| e^{[\mu-3 \epsilon-2(2 n-1) b] t} .
$$

Choose $\epsilon$ subject to

$$
0<3 \epsilon \leqq \mu-2(2 n-1) b ;
$$

then $\mu-3 \epsilon-2(2 n-1) b \geqq 0$ and, in consequence of the first relation (3.13), we have

$$
\lim e^{(\mu-e) t} v=\infty .
$$

This is contrary to the relation $\mu=C(v \mid \exp t)$. Hence (3.14) is impossible and one has

$$
\mu \leqq 2(2 n-1) b .
$$

It may be similarly shown that $\mu \geqq-2(2 n-1) b$. Thus 


$$
-2(2 n-1) b \leqq C\left(x_{1}^{2}+\cdots+x_{n}^{2} \mid e^{t}\right) \leqq 2(2 n-1) b,
$$

and, by virtue of Lemma 3.2,

$$
-(2 n-1) b \leqq C\left(x_{1}, \cdots, x_{n} \mid e^{t}\right) \leqq(2 n-1) b .
$$

We have obtained the following result.

THEOREM 3.1. If in the differential system (3.1) the coefficients are continuous and

$$
\left|p_{i, j}(t)\right| \leqq b<\infty \quad\left(i, j=1, \cdots, n ; t \geqq t_{0}\right),
$$

the following may be asserted for every solution, $x_{j}(j=1, \cdots, n)$, of (3.1). On writing $v=x_{1}^{2}+\cdots+x_{n}^{2}$, we have (3.15), (3.15a) and, more generally, (3.13).

Consider now the case when $\lim p_{i, j}(t)=0$. Let $m(t)$ be the "least" monotone non-increasing continuous function such that

$$
\left|p_{i, j}(t)\right| \leqq m(t) \quad(i, j=1, \cdots, n) .
$$

Existence of such a function is inferred without difficulty. Necessarily $\lim m(t)=0$. Moreover, inasmuch as we exclude the case, trivial from our point of view, when the $p_{i, j}(t)$ are identically zero, we have

$$
m(t)>0 \text {. }
$$

Applying Theorem 3.1 (in particular (3.13)) to the interval $(\tau, \infty)$, where $\tau \geqq t_{0}$, it is concluded that

$$
\lim _{t \rightarrow \infty} e^{(\sigma(\tau)+\epsilon) t} v=\infty, \quad \lim _{t \rightarrow \infty} e^{-(\sigma(\tau)+\epsilon) t} v=0 \quad(\text { all } \epsilon>0),
$$

with

$$
\sigma(\tau)=2(2 n-1) m(\tau) .
$$

In fact, it is noted that for the interval $(\tau \leqq t<\infty)$ one may take $b=m(\tau)$.

In view of (3.16) there exists a number $\epsilon_{0}(>0)$ so that the equation

$$
\sigma(\tau)=\epsilon \quad\left(0<\epsilon \leqq \epsilon_{0}\right)
$$

has a solution $\tau(\epsilon)$; necessarily

$$
\sigma(\tau) \leqq \epsilon \quad \text { (for } \tau \geqq \tau(\epsilon)),
$$

inasmuch as $\sigma(\tau)$ of (3.17a) is monotone non-increasing just as $m(\tau)$ is. Thus, given $\epsilon$ such that $0<\epsilon \leqq \epsilon_{0}$, in (3.17) one may put $\tau=\tau(\epsilon)$, which will yield

$$
\lim _{t \rightarrow \infty} e^{2 \epsilon t} v=\infty, \quad \lim _{t \rightarrow \infty} e^{-2 \epsilon t} v=0 .
$$

Whence

$$
C_{s}\left(v \mid e^{t}\right)=0
$$

We have obtained 
THEOREM 3.2. Let the coefficients of the differential system (3.1) be continuous and

$$
\lim _{t \rightarrow \infty} p_{i, j}(t)=0 \quad(i, j=1, \cdots, n) .
$$

For every solution $x_{1}, \cdots, x_{n}$ of (3.1) we shall have (3.18), where $v=x_{1}^{2}+\cdots+x_{n}^{2}$.

This Theorem is applicable to the general system (3.1) envisaged at the beginning of this section. In fact, there are two cases at hand:

Case 3.19. The integral

$$
\int_{t_{0}}^{\infty} p(t) d t \quad(p(t) \text { from }(3.2))
$$

diverges.

Case 3.20. The above integral converges. That is, $p(t) \rightarrow 0$ sufficiently fast, as $t \rightarrow+\infty$.

Consider the Case 3.19 and let $\rho(t)$ be a continuous function such that

$$
\rho(t) \geqq p(t) \text {. }
$$

Then the integral

$$
\int_{t_{0}}^{\infty} \rho(t) d t
$$

will diverge. Application of the transformation

$$
\tau=\int_{t_{0}}^{t} \rho(t) d t
$$

to (3.1) will yield

$$
\frac{d x_{i}}{d t}=q_{1, i}(\tau) x_{1}+\cdots+q_{n, i}(\tau) x_{n} \quad(i=1, \cdots, n)
$$

with

$$
q_{i, j}(\tau)=\frac{p_{i, j}(t)}{\rho(t)}
$$

The $q_{i, j}(\tau)$ will be continuous for $\tau \geqq 0$ and

$$
\left|q_{i, j}(\tau)\right| \leqq \frac{p(t)}{\rho(t)}=r(\tau) \leqq 1 \quad(i, j=1, \cdots, n ; \tau \geqq 0) .
$$

If $\rho(t)$ is so chosen that

$$
\lim _{t \rightarrow+\infty} \frac{p(t)}{\rho(t)}=0
$$


one will have

$$
\lim _{\tau \rightarrow \infty} r(\tau)=0
$$

and, in view of (3.23a), Theorem 3.2 will be applicable to the system (3.23), yielding the relation

$$
C_{8}\left(x_{1}^{2}+\cdots+x_{n}^{2} \mid e^{\tau}\right)=0
$$

for every solution $x_{1}, \cdots, x_{n}$ of (3.23). By virtue of (3.22) this will imply that in the Case 3.19 for every solution $x_{1}, \cdots, x_{n}$ of the differential system (3.1) we have

$$
C_{s}\left(x_{1}^{2}+\cdots+x_{n}^{2} \mid \exp \left\{\int_{t_{0}}^{t} \rho(t) d t\right\}\right)=0,
$$

provided $\rho(t)$ satisfies (3.21), (3.24).

The degree of precision afforded by an equality (3.25) varies according to the choice of $\rho(t)$ (subject to (3.21), (3.24))( $\left.{ }^{6}\right)$. If $\rho_{1}(t)$ and $\rho_{2}(t)$ are two functions, subject to (3.21), (3.24) and satisfying $\rho_{1}(t) \leqq \rho_{2}(t)$, of the two statements

$$
C_{s}\left(v \mid \exp \left\{\int_{t_{0}}^{t} \rho_{1}(t) d t\right\}\right)=0, \quad C_{s}\left(v \mid \exp \left\{\int_{t_{0}}^{t} \rho_{2}(t) d t\right\}\right)=0
$$

the first one will be as precise as or more precise than the second. Clearly there exists no particular function $\rho(t)$, satisf ying (3.21) and (3.24), for which (3.25) represents the most precise result.

Consider now Case 3.20. Theorem 3.2 will be applicable directly to the system (3.1). Let $\rho(t)$ be positive and continuous (for $t \geqq t_{0}$ ) and let $\rho(t) \rightarrow 0$ (as $t \rightarrow \infty$ ) sufficiently slowly so that

$$
\lim \frac{p(t)}{\rho(t)}=0, \quad \int_{t_{0}}^{\infty} \rho(t) d t \text { diverges. }
$$

Applying to the system (3.1) the transformation (3.22), we repeat the steps leading from (3.22) to (3.24a). Thus, in the Case 3.20 (and, more generally, when $\left.\lim p_{i, j}(t)=0\right)$ for every solution $x_{1}, \cdots, x_{n}$ of the differential system (3.1) we have

$$
C_{s}\left(x_{1}^{2}+\cdots+x_{n}^{2} \mid \exp \left\{\int_{t_{0}}^{t} \rho(t) d t\right\}\right)=0,
$$

where $\rho(t)(>0)$ is taken continuous (for $t \geqq t_{0}$ ) and subject to (3.26). With respect to the degree of precision presented by the above result a statement may be made analogous to that for the Case 3.19.

( $)$ If one takes $\rho(t)>0$, the condition (3.21) will be non-essential. 
The following is a simple application of the above result. Suppose

$$
\left|p_{i, j}(t)\right| \leqq a t^{-\alpha} \quad\left(a>0, \alpha>1 ; t \geqq t_{0}>0\right) ;
$$

on taking $\rho(t)=t^{-1}$ we then obtain

$$
C_{8}\left(x_{1}^{2}+\cdots+x_{n}^{2} \mid t / t_{0}\right)=0
$$

for every solution $x_{1}, \cdots, x_{n}$ of (3.1).

4. Growth of solutions for linear systems. In the previous section we obtained results relating to properties of "growth," at $t=+\infty$, of solutions of linear systems (3.1). We shall now apply a different method, based on "product integration," for the purpose of establishing some further results regarding properties of solutions of (3.1) for $t$ large.

Using matrix notation the system (3.1) may be written as

$$
X^{(1)}(t)=X(t) P(t), \quad X(t)=\left(x_{i, j}(t)\right), \quad P(t)=\left(p_{i, j}(t)\right),
$$

$i, j=1, \cdots, n$. Here and in the sequel the element $a_{i, j}$ displayed in a matrix $\left(a_{i, j}\right)$ will be the one in the $i$ th row and $j$ th column. If $X(t)$ satisfies $(4.1)$, the elements of any row in $X(t)$ will constitute a solution of (3.1). With $t>t_{0}$, we subdivide the interval $\left(t_{0}, t\right)$ into $N$ equal subintervals and call their end points

$$
t_{\nu}=t_{0}+\nu \Delta \quad(\nu=0,1, \cdots, N), \quad t=t_{N},
$$

where

$$
\Delta=\frac{t-t_{0}}{N}
$$

We then form the matrix

$$
X_{N}=\left(x_{N: i, j}\right)=\left(I+\Delta P\left(t_{0}\right)\right)\left(I+\Delta P\left(t_{1}\right)\right) \cdots\left(I+\Delta P\left(t_{N-1}\right)\right),
$$

where $I=\left(\delta_{i, j}\right)$ is the identity matrix. In consequence of the known theory of "product integration" the limit

$$
\lim _{N \rightarrow \infty} X_{N}=X(t)=\left(x_{i, j}(t)\right)
$$

exists for all $t \geqq t_{0}$ and the matrix $X(t)$ will satisfy (4.1); moreover, $X\left(t_{0}\right)=I$. We have taken equal subintervals for convenience.

By (4.3)

$$
\begin{aligned}
X_{N}=I & +\Delta \sum_{\nu_{1}=0}^{N-1} P\left(t_{\nu_{1}}\right)+\Delta^{2} \sum_{\nu_{1}<\nu_{2}} P\left(t_{\nu_{1}}\right) P\left(t_{\nu_{2}}\right)+\cdots \\
& +\Delta^{m} \sum_{\nu_{1}<\cdots<\nu_{m}} P\left(t_{\nu_{1}}\right) P\left(t_{\nu_{2}}\right) \cdots P\left(t_{\nu_{m}}\right)+\cdots+\Delta^{N} P\left(t_{0}\right) P\left(t_{1}\right) \cdots P\left(t_{N-1}\right) ;
\end{aligned}
$$

here $\nu_{j}=0,1, \cdots, N-1$. Thus 


$$
X_{N}-I=\sum_{m=1}^{N} \Delta^{m} L_{m}, \quad L_{m}=\left(l_{m: i, j}\right),
$$

where the element $l_{m: i, j}$ in the matrix $L_{m}$ is of the form

$$
l_{m: i, j}=\text { element in the } i \text { th row and } j \text { th }
$$

$$
\text { column of } \sum_{v_{1}<\cdots<v_{m}} P\left(t_{v_{1}}\right) \cdots P\left(t_{v_{m}}\right)
$$

$$
=\sum_{v_{1}<\cdots<v_{m}} \sum_{\tau_{1}, \ldots, \tau_{m-1}=1}^{n} p_{i, \tau_{1}}\left(t_{v_{1}}\right) p_{\tau_{1}, \tau_{2}}\left(t_{v_{2}}\right) \cdots p_{\tau_{m-1}, j}\left(t_{v_{m}}\right) .
$$

Let $p(t)$ be a continuous function such that

$$
\left|p_{i, j}(t)\right| \leqq p(t)
$$$$
(i, j=1, \cdots, n)
$$

In consequence of $(4.5 \mathrm{a})$ it is concluded that

$$
\begin{aligned}
\left|\Delta^{m} l_{m: i, j}\right| & \leqq \sum_{\nu_{1}<\cdots<v_{m}} \sum_{\tau_{1}, \cdots, \tau_{m-1}=1}^{n}\left(\Delta p\left(t_{\nu_{1}}\right)\right)\left(\Delta p\left(t_{\nu_{2}}\right)\right) \cdots\left(\Delta p\left(t_{\nu_{m}}\right)\right) \\
& =\sum_{\nu_{1}, \cdots}\left(\Delta p\left(t_{\nu_{1}}\right)\right) \cdots\left(\Delta p\left(t_{v_{m}}\right)\right) \sum_{\tau_{1}, \cdots} 1 \\
& =\sum_{\nu_{1}<\cdots<v_{m}}\left(\Delta p\left(t_{v_{1}}\right)\right) \cdots\left(\Delta p\left(t_{\nu_{m}}\right)\right) n^{m-1} \\
& =\frac{1}{n} \sum_{\nu_{1}<\cdots<v_{m}}\left(n \Delta p\left(t_{\nu_{1}}\right)\right)\left(n \Delta p\left(t_{\nu_{2}}\right)\right) \cdots\left(n \Delta p\left(t_{\nu_{m}}\right)\right) .
\end{aligned}
$$

By (4.5) and (4.7)

$$
\begin{aligned}
n\left|x_{N: i, j}-\delta_{i, j}\right| & \leqq n \sum_{m=1}^{N}\left|\Delta^{m} l_{m: i, j}\right| \\
& \leqq \sum_{m=1}^{N} \sum_{\nu_{1}<\cdots<\nu_{m}}\left(n \Delta p\left(t_{\nu_{1}}\right)\right)\left(n \Delta p\left(t_{\nu_{2}}\right)\right) \cdots\left(n \Delta p\left(t_{v_{m}}\right)\right),
\end{aligned}
$$

where $\nu_{j}=0, \cdots, N-1$. With the aid of the identity

$$
-1+\left(1+c_{0}\right)\left(1+c_{1}\right) \cdots\left(1+c_{N-1}\right)=\sum_{m=1}^{N} \sum_{\nu_{1}<\cdots<\nu_{m}} c_{\nu_{1}} c_{\nu_{2}} \cdots c_{\nu_{m}}
$$

$\left(\nu_{j}=0, \cdots, N-1\right)$ from (4.8) we infer

$$
n\left|x_{N: i, j}-\delta_{i, j}\right| \leqq-1+\prod_{r=0}^{N-1}\left(1+n \Delta p\left(t_{n}\right)\right) .
$$

Now, inasmuch as $n \Delta p\left(t_{n}\right) \geqq 0$ and since

$$
1+u \leqq e^{u}
$$

(for $u \geqq 0$ ),

we obtain 


$$
n\left|x_{N: i, j}-\delta_{i, j}\right| \leqq-1+\exp n \sum_{r=0}^{N-1} \Delta p\left(t_{r}\right) .
$$

From this in the limit, as $N \rightarrow \infty$, it is deduced that

$$
\left|x_{i, j}(t)-\delta_{i, j}\right| \leqq \frac{1}{n}\left[-1+\exp \left\{n \int_{t_{0}}^{t} p(t) d t\right\}\right]=\alpha(t)
$$

here the $x_{i, j}(t)(i, j=1, \cdots, n)$ are the elements of the matrix (4.4). The best result is presented by (4.9) when $p(t)$ of $(4.6)$ is taken "least."

Let

$$
t_{0} \leqq t \leqq \tau
$$

We subdivide the interval $(t, \tau)$ into $N$ equal subintervals, with end points given by

$$
t=\tau_{N}<\tau_{N-1}<\cdots<\tau_{1}<\tau_{0}=\tau,
$$

where

$$
\tau_{\nu}=\tau+\nu \delta, \quad \delta=\frac{t-\tau}{N}=-|\delta|
$$

If the matrix

$$
Z_{N}=\left(z_{N ; i, j}\right)=\left(I+\delta P\left(\tau_{0}\right)\right)\left(I+\delta P\left(\tau_{1}\right)\right) \cdots\left(I+\delta P\left(\tau_{N-1}\right)\right)
$$

is formed, in consequence of the theory of product integration it is deduced that the limit

$$
\lim _{N} Z_{N}=Z(t)=\left(z_{i, j}(t)\right) \quad\left(t_{0} \leqq t \leqq \tau\right)
$$

exists and satisfies (4.1); we have

$$
Z(\tau)=I \text {. }
$$

In place of (4.8) we now have inequalities obtained by replacing

$$
x_{N: i, j}, t_{v}, \Delta
$$

by

$$
z_{N: i, j}, \tau_{\nu},-\delta,
$$

respectively. We had $\Delta>0$, but now $\delta<0$. Accordingly, in place of $(4.8 \mathrm{a})$ it is established that

$$
n\left|z_{N: i, j}-\delta_{i, j}\right| \leqq-1+\exp \left\{-n \sum_{r=0}^{N-1} \delta p\left(\tau_{r}\right)\right\} .
$$


In the limit, as $N \rightarrow \infty$, this will yield the following inequalities for the functions $z_{i, j}(t)$ of $(4.11 \mathrm{a})$

$$
n\left|z_{i, j}(t)-\delta_{i, j}\right| \leqq-1+\exp \left\{-n \int_{\tau}^{t} p(t) d t\right\} \quad\left(t_{0} \leqq t \leqq \tau\right) .
$$

When the integral

$$
\int_{t_{0}}^{\infty} p(t) d t
$$

converges, the following is observed. The matrix

$$
X(t)=\left(x_{i, j}(t)\right)=\lim _{\tau \rightarrow+\infty} Z(t)
$$

satisfies the system (4.1); moreover,

$$
X(+\infty)=I
$$

and, by (4.12),

$$
\begin{aligned}
\left|x_{i, j}(t)-\delta_{i, j}\right| \leqq \frac{1}{n}\left[-1+\exp \left\{n \int_{t}^{\infty} p(t) d t\right\}\right]= & \beta(t) \\
& \left(t_{0} \leqq t<\infty\right) .
\end{aligned}
$$

Accordingly one may formulate the theorem.

THEOREM 4.1. The following may be stated for the solutions of the differential system (3.1), whose equivalent matrix form is (4.1).

Let $p(t)$ be a continuous function such that

$$
\left|p_{i, j}(t)\right| \leqq p(t)
$$

The matrix solution $X(t)=\left(x_{i, j}(t)\right)$, for which $X\left(t_{0}\right)=I$, satisfies the inequalities (4.9). In the case when the integral

$$
\int_{t_{0}}^{\infty} p(t) d t
$$

converges, there exists a matrix solution $X(t)$, for which $X(+\infty)=I$; the elements of $X(t)$ will satisfy the inequalities (4.14).

It is noted that, when (4.13) converges, the function $\alpha(t)$ of (4.9) is uniformly bounded; in fact

$$
\alpha(t) \leqq \alpha(\infty)<\infty \quad\left(t_{0} \leqq t<\infty\right) .
$$

When (4.13) diverges $\alpha(t)$ is monotone increasing and

$$
\lim _{t \rightarrow \infty} \alpha(t)=+\infty ;
$$


moreover, in this case, the faster

$$
\phi(t)=\int_{t_{0}}^{t} p(t) d t
$$

approaches $+\infty$, as $t \rightarrow \infty$, the faster will $\alpha(t)$ approach $+\infty$ with $t$.

When (4.13) converges, the function $\beta(t)$ of (4.14) will approach zero, as $t \rightarrow+\infty$. In this case, the faster $p(t) \rightarrow 0$ (as $t \rightarrow \infty)$, the faster will $\beta(t)$ approach zero with $1 / t$.

Comparison of the results of Theorems 4.1, 3.1 leads to the conclusion that the two theorems overlap and, in a sense, supplement each other.

Corollary 4.1. With $p(t)$ from (4.6) and

$$
\int_{t_{0}}^{\infty} p(t) d t
$$

convergent, every solution $Y(t)=\left(y_{i, j}(t)\right)$ (defined for $\left.t \geqq t_{0}\right)$ of (4.1) has the properties:

$$
\lim Y(t)=C=\left(c_{i, j}\right)
$$$$
(\text { as } t \rightarrow \infty),
$$

where the $c_{i, j}$ are constants;

$$
\left|y_{i, j}(t)-c_{i, j}\right| \leqq n c \beta(t),
$$

with $\beta(t)$ from (4.14) and the constant $c$ from the inequalities

$$
\left|c_{i, j}\right| \leqq c
$$

$(i, j=1, \cdots, n)$.

In fact,

$$
Y(t)=C X(t), \quad C=\left(c_{i, j}\right),
$$

where the $c_{i, j}$ are some constants and $X(t)$ is a matrix solution of (4.1) for which $X(+\infty)=I$. The solution $X(t)$, with the stated property, exists in consequence of Theorem 4.1. The relation (4.15) follows at once. Now

$$
Y(t)-C=\left(y_{i, j}(t)-c_{i, j}\right)=C(X(t)-I)
$$

and

$$
y_{i, j}(t)-c_{i, j}=\sum_{\tau=1}^{n} c_{i, \tau}\left(x_{\tau, j}(t)-\delta_{\tau, j}\right) .
$$

Thus, by (4.15a) and (4.14)

$$
\left|y_{i, j}(t)-c_{i, j}\right| \leqq n c \beta(t),
$$

which establishes the corollary.

Let us consider the case when there exists a continuous function $\rho(t)$ such that 


$$
p_{i, j}(t) \geqq \rho(t) \geqq 0
$$

$(i, j=1, \cdots, n)$.

We repeat the developments leading up to (4.5a). By (4.5a) and (4.16)

$$
\Delta l_{1: i, j}=\sum_{\nu_{1}=0}^{N-1} \Delta p_{i, j}\left(t_{\nu_{1}}\right) \geqq \sum_{\nu_{1}=0}^{N-1} \Delta p\left(t_{\nu_{1}}\right), \quad \Delta^{m} l_{m: i, j} \geqq 0 .
$$

Whence, in view of (4.5),

$$
x_{N: i, j}-\delta_{i, j}=\Delta l_{1: i, j}+\sum_{m=2}^{N} \Delta^{m} l_{m: i, j} \geqq \Delta l_{1: i, j} \geqq \sum_{\nu_{1}=0}^{N-1} \Delta \rho\left(t_{\nu_{1}}\right)
$$

and, in the limit (as $N \rightarrow \infty$ ),

$$
x_{i, j}(t)-\delta_{i, j} \geqq \lim _{N} \sum_{\nu_{1}=0}^{N-1} \Delta p\left(t_{\nu_{1}}\right)=\int_{t_{0}}^{t} \rho(t) d t .
$$

Hence we may state the theorem.

THEOREM 4.2. Suppose (4.16) holds. Then the $x_{i, j}(t)$ of the matrix solution $X(t)=\left(x_{i, j}(t)\right)$ of (4.1), with $X\left(t_{0}\right)=I$, will satisfy the inequalities (4.17).

CoRollary 4.2. Suppose that the coefficients in (4.1) satisfy

$$
p_{i, j}(t) \leqq-\rho(t)<0 \quad(i, j=1, \cdots, n),
$$

where $\rho(t)$ is continuous. With $X(t)=\left(x_{i, j}(t)\right)$ denoting a solution of (4.1), reducing to $I$ for $t_{0}$, the elements $\bar{x}_{i, j}(t)$ of the inverse of $X(t)$ will satisfy the inequalities

$$
\bar{x}_{i, j}(t) \geqq \delta_{i, j}+\int_{t_{0}}^{t} \rho(t) d t \quad(i, j=1, \cdots, n) .
$$

The corollary is established on noting that

$$
\bar{X}(t)=\left(\bar{x}_{i, j}(t)\right)
$$

satisfies

$$
\bar{X}^{(1)}(t)=-P(t) \bar{X}(t),
$$

whenever $X(t)$ is a matrix solution of (4.1).

Further results will be obtained with the aid of product integration and of the identity

$$
\begin{aligned}
& h_{i, \tau_{1}}\left(t_{\nu_{1}}\right)+h_{\tau_{1}, \tau_{2}}\left(t_{\nu_{2}}\right)+\cdots+h_{\tau_{m-2}, \tau_{m-1}}\left(t_{v_{m-1}}\right)+h_{\tau_{m-1}, j}\left(t_{v_{m}}\right) \\
& =h_{i, j}(t)+\left[h_{j, i}(t)-h_{j, i}\left(t_{v_{m}}\right)\right]+\left[h_{\tau_{m-1}, i}\left(t_{\nu_{m}}\right)-h_{\tau_{m-1}, i}\left(t_{\nu_{m-1}}\right)\right]+\cdots \\
& \quad+\left[h_{\tau_{2}, i}\left(t_{\nu_{3}}\right)-h_{\tau_{2}, i}\left(t_{\nu_{2}}\right)\right]+\left[h_{\tau_{1}, i}\left(t_{\nu_{2}}\right)-h_{\tau_{1}, i}\left(t_{\nu_{1}}\right)\right]
\end{aligned}
$$

where we have put
(4.18a)
$h_{i, j}(t)=h_{i}(t)-h_{j}(t)$
$(i, j=1, \cdots, n)$. 
Suppose the $h_{i}(t)$ are continuous for $t \geqq t_{0}$ and the $h_{i}^{(1)}(t)(i=1, \cdots, n)$ exist. If we have

$$
h_{1}^{(1)}(t) \geqq h_{2}^{(1)}(t) \geqq \cdots \geqq h_{n}^{(1)}(t)
$$

then

$$
h_{\tau, i}^{(1)}(t)=h_{\tau}^{(1)}(t)-h_{i}^{(1)}(t) \leqq 0
$$$$
(i=1, \cdots, \mu ; \tau=1, \cdots, n)
$$

where $\mu$ is from the inequalities

$$
h_{1}^{(1)}(t)=h_{2}^{(1)}(t)=\cdots=h_{\mu}^{(1)}(t) \geqq h_{\mu+1}^{(1)}(t) \geqq \cdots .
$$

Thus, under (4.19), $1 \leqq \mu \leqq n$. With $t_{0} \leqq t^{\prime} \leqq t^{\prime \prime}$, from (4.19a) one would obtain (4.19c) $h_{\tau, i}\left(t^{\prime \prime}\right)-h_{\tau, i}\left(t^{\prime}\right)=\int_{t^{\prime}}^{t^{\prime \prime}} h_{\tau, i}^{(1)}(t) d t \leqq 0 \quad(i=1, \cdots, \mu ; \tau=1, \cdots, n)$.

Whence, if (4.19) (see (4.19b)) holds, the identity (4.18) will yield

$$
\begin{array}{r}
h_{i, \tau_{1}}\left(t_{v_{1}}\right)+h_{\tau_{1}, \tau_{2}}\left(t_{\nu_{2}}\right)+\cdots+h_{\tau_{m-2}, \tau_{m-1}}\left(t_{\nu_{m-1}}\right)+h_{\tau_{m-1}, j}\left(t_{v_{m}}\right) \leqq h_{i, j}(t) \\
(i=1, \cdots, \mu ; j=1, \cdots, n),
\end{array}
$$

whenever

$$
t_{0} \leqq t_{v_{1}} \leqq t_{v_{2}} \leqq \cdots \leqq t_{v_{m}} \leqq t .
$$

If instead of (4.19) we have

$$
h_{1}^{(1)}(t) \leqq h_{2}^{(1)}(t) \leqq \cdots \leqq h_{n}^{(1)}(t),
$$

then for some number $\eta$, such that $1 \leqq \eta \leqq n$, the inequalities

$$
h_{1}^{(1)}(t) \leqq \cdots \leqq h_{\eta}^{(1)}(t)=h_{\eta+1}^{(1)}(t)=\cdots=h_{n}^{(1)}(t)
$$

will hold. It will follow that

$$
h_{r, i}^{(1)}(t) \leqq 0 \quad(i=\eta, \eta+1, \cdots, n ; \tau=1, \cdots, n) .
$$

This, in turn, would imply

$$
h_{\tau, i}\left(t^{\prime \prime}\right)-h_{\tau, i}\left(t^{\prime}\right) \leqq 0 \quad(i=\eta, \eta+1, \cdots, n ; \tau=1, \cdots, n)
$$

for any values $t^{\prime}, t^{\prime \prime}$ such that $t_{0} \leqq t^{\prime} \leqq t^{\prime \prime}$. Hence, on taking note of (4.18), it is observed that, if (4.21) (see (4.21a)) holds, one has

$$
\begin{array}{r}
h_{i, \tau_{1}}\left(t_{v_{1}}\right)+h_{\tau_{1}, \tau_{2}}\left(t_{v_{2}}\right)+\cdots+h_{\tau_{m-2}, \tau_{m-1}}\left(t_{v_{m-1}}\right)+h_{\tau_{m-1}, j}\left(t_{v_{m}}\right) \leqq h_{i, j}(t) \\
(i=\eta, \eta+1, \cdots, n ; j=1, \cdots, n),
\end{array}
$$

whenever (4.20a) is satisfied.

Let $w(t), h_{j}(t)(j=1, \cdots, n)$ be functions such that (4.19) holds and 


$$
\left|p_{i, j}(t)\right| \leqq w(t) e^{h_{i}(t)-h_{j}(t)}
$$

$(i, j=1, \cdots, n)$,

the function $w(t)(\geqq 0)$ being continuous for $t \geqq t_{0}$. Such functions exist in all cases. Repeating the developments leading to $(4.5 \mathrm{a})$ we now obtain

$$
\begin{aligned}
&\left|\Delta^{m} l_{m: i, j}\right| \leqq \sum_{p_{1}<\cdots<v_{m}} \sum_{\tau_{1}, \cdots, \tau_{m-1}=1}^{n}\left(\Delta w\left(t_{\nu_{1}}\right)\right)\left(\Delta w\left(t_{\nu_{2}}\right)\right) \cdots\left(\Delta w\left(t_{v_{m}}\right)\right) \\
& \cdot \exp \left[h_{i, \tau_{1}}\left(t_{\nu_{1}}\right)+h_{\tau_{1}, \tau_{2}}\left(t_{\nu_{2}}\right)+\cdots+h_{\tau_{m-2}, \tau_{m-1}}\left(t_{v_{m-1}}\right)+h_{\tau_{m-1}, j}\left(t_{v_{m}}\right)\right] .
\end{aligned}
$$

Inasmuch as $(4.20 \mathrm{a})$ is satisfied, application of $(4.20)$ to (4.24) is possible, yielding

(4.24a) $\quad\left|\Delta^{m} l_{m: i, j}\right| \leqq e^{h_{i, j}(t)} \sum_{\nu_{1}<\cdots<v_{m}} \sum_{\tau_{1}, \cdots}\left(\Delta w\left(t_{\nu_{l}}\right)\right) \cdots\left(\Delta w\left(t_{v_{m}}\right)\right)$

for $i=1, \cdots, \mu$ ( $\mu$ from (4.19b)). Using the steps employed in (4.7), from $(4.24 \mathrm{a})$ it is deduced that

$$
\left|\Delta^{m} l_{m: i, j}\right| \leqq e^{h_{i, j}(t)} \frac{1}{n} \sum_{\nu_{1}<\cdots<v_{m}}\left(n \Delta w\left(t_{v_{1}}\right)\right) \cdots\left(n \Delta w\left(t_{v_{m}}\right)\right)
$$

$\left(i=1, \cdots, \mu ; j=1, \cdots, n ; 0 \leqq \nu_{\sigma} \leqq N-1\right)$. By (4.5) and the above (see (4.8)) one has

$$
n\left|x_{N: i, j}-\delta_{i, j}\right| e^{-h_{i, j}(t)} \leqq \sum_{m=1}^{N} \sum_{\nu_{1}, \ldots}\left(n \Delta w\left(t_{\nu_{1}}\right)\right) \cdots\left(n \Delta w\left(t_{v_{m}}\right)\right)
$$

$(i=1, \cdots, \mu)$ and, with the aid of the identity subsequent to (4.8), we find

$$
n\left|x_{N: i, j}-\delta_{i, j}\right| e^{-h_{i, j}(t)} \leqq-1+\prod_{r=0}^{N-1}\left(1+n \Delta w\left(t_{r}\right)\right) \quad(i=1, \cdots, \mu) .
$$

Thus

$$
n\left|x_{N: i, j}-\delta_{i, j}\right| e^{-h_{i, j}(t)} \leqq-1+\exp n \sum_{r=0}^{N-1} \Delta w\left(t_{r}\right) \quad(i=1, \cdots, \mu),
$$

and, in the limit (as $N \rightarrow \infty$ ),

$$
\begin{array}{r}
\left|x_{i, j}(t)-\delta_{i, j}\right| \leqq \frac{1}{n} e^{h_{i}(t)-h_{j}(t)}\left[-1+\exp \left\{n \int_{t_{0}}^{t} w(t) d t\right\}\right] \\
(i=1, \cdots, \mu ; j=1, \cdots, n) .
\end{array}
$$

Let continuous $w(t)(\geqq 0)$ and the $h_{j}(t)$ be such that $(4.21)$ holds and the inequalities (4.23) are satisfied. On making use of (4.22), we now obtain

$$
\begin{aligned}
\left|x_{i, j}(t)-\delta_{i, j}\right| \leqq \frac{1}{n} e^{h_{i}(t)-h_{j}(t)}\left[-1+\exp \left\{n \int_{t_{0}}^{t} w(t) d t\right\}\right] & (i=\eta, \eta+1, \cdots, n ; j=1, \cdots, n),
\end{aligned}
$$


where $\eta$ is from (4.21a). As in (4.25) the $x_{i, j}(t)$ are the elements of the matrix $X(t)$, satisfying the system $(4.1) ; X\left(t_{0}\right)=I$.

Inequalities (4.25) refer to the first $\mu$ rows of $X(t)$, while (4.26) refers to the last $n-\eta+1$ rows. We recall that the elements in any row of $X(t)$ constitute a solution of the system (3.1). The $h_{j}(t)$ in (4.25) and (4.26) do not need to be the same.

Inequalities of the form (4.23) imply, in particular, that

$$
w(t) \geqq\left|p_{i, i}(t)\right| \quad(i=1, \cdots, n) .
$$

Thus, it is not always possible to choose $w(t)$ so that the integral

$$
\int_{t_{0}}^{\infty} w(t) d t
$$

converges. However, for some systems (4.1) one may choose $w(t)$ so that (4.27) converges. Suppose now that the latter is the case. Let $w(t)$ be a continuous function such that (4.27) converges, while

$$
\left|p_{i, j}(t)\right| \leqq w(t) e^{h_{i}(t)-h_{j}(t)} \quad(i, j=1, \cdots, n),
$$

where the $h_{j}(t)$ are such that either (4.19) or (4.21) holds. We recall the notation introduced in connection with (4.10)-(4.10a), as well as the subsequent developments leading to (4.14). The formula corresponding to (4.8) will be

$$
n\left|z_{N: i, j}-\delta_{i, j}\right| \leqq n \sum_{m=1}^{N}(-\delta)^{m}\left|l_{m: i, j}\right| \text {. }
$$

On the other hand, making the replacements indicated subsequent to (4.11a), in view of $(4.5 \mathrm{a})$ it is concluded that

$$
\begin{aligned}
(-\delta)^{m} \mid & l_{m: i, j} \mid \\
& \leqq \sum_{\nu_{1}<\cdots<\nu_{m}} \sum_{\lambda_{1}, \cdots, \lambda_{m-1}=1}^{n}\left(-\delta p_{i, \lambda_{1}}\left(\tau_{\nu_{1}}\right)\right)\left(-\delta p_{\lambda_{1}, \lambda_{2}}\left(\tau_{\nu_{2}}\right)\right) \cdots\left(-\delta p_{\lambda_{m-1}, j}\left(\tau_{\nu_{m}}\right)\right)
\end{aligned}
$$

$\left(0 \leqq \nu_{\sigma} \leqq N-1\right)$. In consequence of (4.28) this will imply (cf. (4.24))

$$
\begin{aligned}
& (-\delta)^{m}\left|l_{m: i, j}\right| \\
& \quad \sum_{\nu_{1}<\cdots<\nu_{m}} \sum_{\lambda_{1}, \cdots, \lambda_{m-1}=1}^{n}\left(-\delta w\left(\tau_{\nu_{1}}\right)\right)\left(-\delta w\left(\tau_{\nu_{2}}\right)\right) \cdots\left(-\delta w\left(\tau_{\nu_{m}}\right)\right) \\
& \quad \cdot \exp \left[h_{i, \lambda_{1}}\left(\tau_{\nu_{1}}\right)+h_{\lambda_{1}, \lambda_{2}}\left(\tau_{\nu_{2}}\right)+\cdots+h_{\lambda_{m-2}, \lambda_{m-1}}\left(\tau_{\nu_{m-1}}\right)+h_{\lambda_{m-1}, j}\left(\tau_{\nu_{m}}\right)\right]
\end{aligned}
$$

( $\left.i, j=1, \cdots, n ; t<\tau_{\nu_{m}}<\tau_{\nu_{m-1}}<\cdots<\tau_{\nu_{1}} \leqq \tau\right)$. Under (4.19), in place of (4.19c) we obtain

$$
h_{\lambda, i}\left(t^{\prime \prime}\right)-h_{\lambda, i}\left(t^{\prime}\right)=\int_{t^{\prime}}^{t^{\prime \prime}} h_{\lambda, i}^{(1)}(t) d t \leqq 0 \quad\left(i=\eta^{\prime}, \cdots, n ; \lambda=1, \cdots, n\right)
$$


for $t \leqq t^{\prime \prime} \leqq t^{\prime} \leqq \tau$; here $\eta^{\prime}\left(1 \leqq \eta^{\prime} \leqq n\right)$ is, from the inequalities, valid when (4.19) holds-that is,

(4.31a) $\quad h_{1}^{(1)}(t) \geqq h_{2}^{(1)}(t) \geqq \cdots \geqq h_{\eta^{\prime}}^{(1)}(t)=h_{\eta^{\prime}+1}^{(1)}(t)=\cdots=h_{n}^{(1)}(t)$.

On the other hand, under (4.21),

$$
h_{\lambda, i}\left(t^{\prime \prime}\right)-h_{\lambda, i}\left(t^{\prime}\right) \leqq 0
$$$$
\left(i=1, \cdots, \mu^{\prime} ; \lambda=1, \cdots, n\right)
$$

for $t \leqq t^{\prime \prime} \leqq t^{\prime} \leqq \tau$, the integer $\mu^{\prime}$ being from the inequalities

$$
h_{1}^{(1)}(t)=h_{2}^{(1)}(t)=\cdots=h_{\mu^{\prime}}^{(1)}(t) \leqq h_{\mu^{\prime}+1}^{(1)}(t) \leqq \cdots \leqq h_{n}^{(1)}(t) .
$$

By virtue of (4.18)

$$
\begin{aligned}
W= & h_{i, \lambda_{1}}\left(\tau_{\nu_{1}}\right)+h_{\lambda_{1}, \lambda_{2}}\left(\tau_{\nu_{2}}\right)+\cdots+h_{\lambda_{m-1}, j}\left(\tau_{\nu_{m}}\right) \\
= & h_{i, j}(t)+\left[h_{j, i}(t)-h_{j, i}\left(\tau_{\nu_{m}}\right)\right]+\left[h_{\lambda_{m-1}, i}\left(\tau_{\nu_{m}}\right)-h_{\lambda_{m-1}, i}\left(\tau_{\nu_{m-1}}\right)\right]+\cdots \\
& +\left[h_{\lambda_{1}, i}\left(\tau_{\nu_{2}}\right)-h_{\lambda_{1}, i}\left(\tau_{\nu_{1}}\right)\right] .
\end{aligned}
$$

Inasmuch as the $\tau_{j}$ satisfy the inequalities of (4.30), on taking account of the statements in connection with (4.31) and (4.32), the following is inferred. For $j=1, \cdots, n$ and $t_{0} \leqq t \leqq \tau$

$$
W \leqq h_{i, j}(t)\left\{\begin{array}{l}
i=\eta^{\prime}, \cdots, n \text { (under (4.19); cf. (4.31a)), } \\
i=1, \cdots, \mu^{\prime} \quad(\text { under (4.21); cf. (4.32a)) }
\end{array}\right.
$$

Application of (4.34) will yield from (4.30)

$$
(-\delta)^{m}\left|l_{m: i, j}\right| \leqq e^{h_{i, j}(t)} \sum_{\nu_{1}<\cdots<\nu_{m}} \sum_{\lambda_{1}, \cdots}\left(-\delta w\left(\tau_{\nu_{1}}\right)\right) \cdots\left(-\delta w\left(\tau_{\nu_{m}}\right)\right)
$$

for values of $i$ as indicated in (4.34) and for $t_{0} \leqq t \leqq \tau$. Whence, by virtue of (4.29),

$$
\begin{aligned}
n\left|z_{N: i, j}-\delta_{i, j}\right| e^{-h_{i, i}(t)} & \leqq \sum_{m=1}^{N} \sum_{\nu_{1}<\cdots}\left(-n \delta w\left(\tau_{\nu_{1}}\right)\right) \cdots\left(-n \delta w\left(\tau_{\nu_{m}}\right)\right) \\
& =-1+\prod_{r=0}^{N-1}\left(1-n \delta w\left(\tau_{r}\right)\right) \\
& \leqq-1+\exp -n \sum_{r=0}^{N-1} \delta w\left(\tau_{r}\right)
\end{aligned}
$$

for $i=\eta^{\prime}, \cdots, n$, under (4.19), or for $i=1, \cdots, \mu^{\prime}$, under (4.21). Accordingly, in the limit (as $N \rightarrow \infty$ ) one has

$$
\begin{aligned}
&\left|z_{i, j}(t)-\delta_{i, j}\right| \leqq \frac{1}{n} e^{h_{i}(t)-h_{j}(t)}\left[-1+\exp \left\{-n \int_{\tau}^{t} w(t) d t\right\}\right] \\
&\left(i=\eta^{\prime}, \cdots, n \text { or } i=1, \cdots, \mu^{\prime} ; j=1, \cdots, n ; t_{0} \leqq t \leqq \tau\right)
\end{aligned}
$$


The matrix $\left(z_{i, j}(t)\right)$ satisfies the system $(4.1)$; moreover, $\left(z_{i, j}(\tau)\right)=\left(\delta_{i, j}\right)$.

Existence of the limits, as $\tau \rightarrow \infty$, cannot be asserted for all the elements of the matrix $\left(z_{i, j}(t)\right)$. However, the limits

$$
\begin{array}{lr}
\lim _{\tau} z_{i, j}(t)=x_{i, j}(t) & \left(=\eta^{\prime}, \cdots, n ; \text { under }(4.19)\right), \\
\lim _{\tau} z_{i, j}(t)=x_{i, j}(t) & \left(i=1, \cdots, \mu^{\prime} ; \text { under }(4.21)\right)
\end{array}
$$

will exist for $j=1, \cdots, n$; moreover, in consequence of (4.35)

$$
\left|x_{i, j}(t)-\delta_{i, j}\right| \leqq \frac{1}{n} e^{h_{i}(t)-h_{j}(t)}\left[-1+\exp \left\{n \int_{t}^{\infty} w(t) d t\right\}\right]
$$

for values of $i$ as indicated in (4.36), (4.36a), for $j=1, \cdots, n$ and for $t_{0} \leqq t<\infty$, it being supposed that (4.27) converges. For any $i$, for which the above is asserted, the set of $n$ functions

$$
x_{i, 1}(t), x_{i, 2}(t), \cdots, x_{i, n}(t)
$$

constitutes a solution of (3.1).

The above results may be summed as follows.

THEOREM 4.3. Let $w(t)(\geqq 0), h_{j}(t)(j=1, \cdots, n)$ be functions continuous for $t \geqq t_{0}$, such that

$$
\left|p_{i, j}(t)\right| \leqq w(t) \exp \left[h_{i}(t)-h_{j}(t)\right] \quad(i, j=1, \cdots, n),
$$

and such that either (4.19) or (4.21) is satisfied, the $h_{j}(t)$ being derivable.

If $X(t)=\left(x_{i, j}(t)\right)$ is the matrix solution of $(4.1)$, for which $X\left(t_{0}\right)=I$, then

$$
\left|x_{i, j}(t)-\delta_{i, j}\right| \leqq \frac{1}{n} e^{h_{i(t)-h_{j}(t)}}\left[-1+\exp \left\{n \int_{t_{0}}^{t} w(t) d t\right\}\right]
$$

$(j=1, \cdots, n)$ for $i=1, \cdots, \mu$, under (4.19) (cf. (4.19b)), and for $i=\eta, \cdots, n$, under (4.21) (cf. (4.21a)).

Suppose $w(t)$ can be so selected that the integral of $w(t) d t$ extended over $\left(t_{0}, \infty\right)$ converges, while the $h_{j}(t)$ are functions as described above, for which either (4.19) or (4.21) holds. One may then assert that there exists a matrix solution $\left.X(t)=x_{i, j}(t)\right)$ of (4.1) with the following properties. If (4.19) holds, each of the last $n-\eta^{\prime}+1$ rows of $X(t)$ will constitute a solution of the system (3.1). When (4.21) holds, each of the first $\mu^{\prime}$ rows of $X(t)$ will be a solution of (3.1). In this connection, $\eta^{\prime}, \mu^{\prime}$ are from (4.31a) and (4.32a), respectively. The constituent elements $x_{i, j}(t)$ of the solutions of (3.1), just referred to, will satisfy inequalities (4.37) for $j=1, \cdots, n$ and for $t_{0} \leqq t<\infty$.

5. Growth of solutions for nonlinear systems. We consider now nonlinear differential systems of the form important in classical dynamics, 


$$
\frac{d x_{i}}{d t}=f_{i}\left(t ; x_{1}, \cdots, x_{n}\right)=l_{i}+q_{i} \quad(i=1, \cdots, n),
$$

where

$$
l_{i}=p_{1, i}(t) x_{1}+\cdots+p_{n, i}(t) x_{n},
$$

the $p_{s, i}(t)$ being functions of the same type as before, while

$$
q_{i}=\sum_{j_{1}+\cdots+j_{n}=2} p_{i}^{j_{1}, \cdots, j_{n}}(t ; x) x_{1}^{j_{1}} x_{2}^{i_{2}} \cdots x_{n}^{j_{n}} \quad\left(j_{1}, \cdots, j_{n} \geqq 0\right) .
$$

The coefficients in (5.1b) may depend on $(x)=\left(x_{1}, \cdots, x_{n}\right)$ :

$$
p_{i}^{j_{1}, \cdots, j_{n}}(t ; x)=p_{i}^{j_{1}, \cdots, j_{n}}(t)+r_{i}^{j_{1}, \cdots, j_{n}}(t ; x),
$$

where the

$$
p_{i}^{j_{1}, \cdots, j_{n}}(t) \quad\left(j_{1}+\cdots+j_{n}=2 ; j_{1}, \cdots, j_{n} \geqq 0\right)
$$

are independent of $x_{1}, \cdots, x_{n}$ and are continuous for $t \geqq t_{0}$. On the other hand, the

$$
r_{i}^{j_{1}, \cdots, j_{n}}(t ; x)=r_{i}^{j_{1}, \cdots, j_{n}}\left(t ; x_{1}, \cdots, x_{n}\right)
$$

are continuous in $x_{1}, \cdots, x_{n}$, for every $t \geqq t_{0}$, when

$$
t_{0} \leqq t<\infty, \quad\left|x_{j}\right| \leqq H \quad(j=1, \cdots, n ; H>0) ;
$$

moreover, it is assumed that

$$
\left|r_{i}^{j_{1}, \cdots, j_{n}}(t ; x)\right| \leqq r(\|x\|) \leqq r^{0} \quad\left(\|x\|^{2}=x_{1}^{2}+\cdots+x_{n}^{2}\right)
$$

under (5.2). Here $r(u)$ is independent of $i, j_{1}, \cdots, j_{n}$ and $r^{0}$ is independent of $x_{1}, \cdots, x_{n} ;$ furthermore

$$
\lim r(u)=0 \quad(\text { as } u \rightarrow 0) ;
$$

$r^{0}$ may depend on $t$, in which case it is to be continuous for $t \geqq t_{0}$.

Such systems (5.1) will include, in particular, the case when the $f_{i}\left(t ; x_{1}, \cdots, x_{n}\right)$ are analytic in $x_{1}, \cdots, x_{n}$, for $\left|x_{i}\right| \leqq H$ and $t \geqq t_{0}$, and are continuous in $t$, the variables involved being subject to $(5.2) ; f_{i}(t ; 0, \cdots, 0)$ $=0$. In the case when the term "analytic in $x_{1}, \cdots, x_{n}$," above, is replaced by the expression "analytic in $x_{1}, \cdots, x_{n}$ uniformly with respect to $t$ $\left(t \geqq t_{0}\right)$ " ( $\left.{ }^{7}\right)$, one may choose $r(\|x\|)$ and $r^{0}$ in (5.3) independent of $t$.

It is observed that the system

$$
\frac{d x_{i}}{d t}=l_{i} \quad(i=1, \cdots, n)
$$

( $\left.{ }^{7}\right)$ In the sense of Liapounoff. 
is precisely the previously investigated linear differential system (3.1).

Let $p_{0}(t)$ be the "least" function, continuous for $t \geqq t_{0}$ and such that

$$
\left|p_{i, j}(t)\right| \leqq p_{0}(t)
$$

and

$$
\left|p_{i}^{j_{1}, \cdots, j_{n}}(t)\right|+r^{0} \leqq p_{0}(t) .
$$

Inasmuch as the number of continuous functions in the first members of (5.4), (5.4a) is finite, $p_{0}(t)$ may always be chosen as stated above.

The transformation

$$
\tau=\phi(t)=\int_{t_{0}}^{t} p_{0}(t) d t \quad\left(0 \leqq \tau \leqq \phi(+\infty)=\tau^{\prime}\right)
$$

has the properties indicated at the beginning of $\$ 3$. Under (5.5) the system (5.1) takes the form

$$
\frac{d x_{i}}{d \tau}=\bar{f}_{i}=\bar{l}_{i}+\bar{q}_{i} \quad(i=1, \cdots, n),
$$

where

$$
\begin{aligned}
& \bar{l}_{i}=h_{1, i}(\tau) x_{1}+\cdots+h_{n, i}(\tau) x_{n}, \\
& \bar{q}_{i}=\sum_{j_{1}, \cdots} h_{i}^{j_{1}, \cdots, j_{n}}(\tau ; x) x_{1}^{j_{1}} \cdots x_{n}^{j_{n}} \quad\left(j_{1}+\cdots+j_{n}=2\right) .
\end{aligned}
$$

Here

$$
h_{i, j}(\tau)=\frac{p_{i, j}(t)}{p_{0}(t)}, \quad h_{i}^{j_{1}, \cdots, j_{n}}(\tau ; x)=h_{i}^{j_{1}, \cdots, j_{n}}(\tau)+\bar{r}_{i}^{j_{1}, \cdots, j_{n}}(\tau ; x),
$$

with

$$
h_{i}^{j_{1}, \cdots, j_{n}}(\tau)=\frac{1}{p_{0}(t)} p_{i}^{j_{1}, \cdots, j_{n}}(t), \quad \dot{r}_{i}^{j_{1}, \cdots, j_{n}}(\tau ; x)=\frac{1}{p_{0}(t)} r_{i}^{j_{1}, \cdots, j_{n}}(t ; x) .
$$

In consequence of $(5.3)$ and $(5.4 \mathrm{a})$

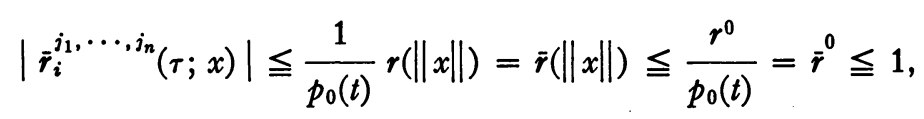

$$
\left|h_{i}^{j_{1}, \cdots, j_{n}}(\tau ; x)\right| \leqq 1, \quad\left|h_{i, j}(\tau)\right| \leqq 1, \quad\left|h_{i}^{j_{1}, \cdots, j_{n}}(\tau)\right| \leqq 1
$$

for $0 \leqq \tau \leqq \tau^{\prime}$ and $\left|x_{j}\right| \leqq H(j=1, \cdots, n)$. It is noted that the function $\bar{r}^{0}$, in (5.6), is continuous in $\tau$ for $0 \leqq \tau \leqq \tau^{\prime}$.

When the integral

$$
\int_{t_{0}}^{\infty} p_{0}(t) d t
$$


diverges, the interval for $\tau$ is $(0,+\infty)$; in the contrary case it is $\left(0, \tau^{\prime}\right)$, where $\tau^{\prime}<+\infty$. In the latter case

$$
p_{0}(t) \leqq b<\infty
$$

and, in view of our purposes, the transformation (5.5) is not needed.

In view of the transformation (5.5) ( $p_{0}(t)$ from (5.4), (5.4a)) there is no loss of generality, if the general nonlinear problem (5.1)-(5.1c) is taken subject to the inequalities

$$
\begin{gathered}
\left|p_{i, j}(t)\right|, \quad\left|p_{i}^{j_{1}, \cdots, j_{n}}(t)\right| \leqq b<\infty \quad\left(j_{1}+\cdots+j_{n}=2\right), \\
\left|p_{i}^{j_{1}, \cdots, j_{n}}(t ; x)\right| \leqq b, \\
\left|r_{i}^{j_{1}, \cdots, j_{n}}(t ; x)\right| \leqq r(\|x\|) \leqq r^{0} \leqq b
\end{gathered}
$$

satisfied for $\left|x_{j}\right| \leqq H(j=1, \cdots, n)$ and $t_{0} \leqq t<\infty, b$ being independent of $t$.

To the system (5.1), under (5.8)-(5.8b), we apply the transformation

$$
z_{i}=x_{i} e^{\lambda t} \quad(\lambda \text { indeterminate). }
$$

The system satisfied by the $z_{i}$ will be of the form

$$
\frac{d z_{i}}{d t}=p_{1, i}(t) z_{1}+\cdots+\left(p_{i, i}(t)+\lambda\right) z_{i}+\cdots+p_{n, i}(t) z_{n}+p_{n, i}^{\prime}
$$

$(i=1, \cdots, n)$, with

$$
p_{n, i}^{\prime}=e^{-\lambda t} \sum_{\nu, \kappa}^{n} p_{i}^{\nu, \kappa} z_{\nu} z_{\kappa}, \quad \delta p_{i}^{\nu, \kappa}=p_{i}^{j_{1}, \cdots, j_{n}}(t)+r_{i}^{j_{1}, \cdots, j_{n}}\left(t ; x_{1}, \cdots, x_{n}\right)
$$

$\left(j_{1}+\cdots+j_{n}=2\right)$, where sets $(\nu, \kappa)$ are in a certain correspondence with sets $\left(j_{1}, \cdots, j_{n}\right)$, while $\delta=1$ for $\nu=\kappa$ and $\delta=2$ for $\nu \neq \kappa$. We write

$$
u=z_{1}^{2}+\cdots+z_{n}^{2} .
$$

Then

$$
\frac{1}{2} \cdot \frac{d u}{d t}=A(\lambda)+B(\lambda)+C(\lambda)
$$

where $A(\lambda), B(\lambda)$ are given by (3.9a) and

$$
C(\lambda)=\sum_{i=1}^{n} z_{i} p_{n, i}^{\prime}
$$

Using the inequalities (5.8), from the developments of $\$ 3$ we infer that for all $t \geqq t_{0}$. 


$$
\begin{array}{lr}
A(\lambda)+B(\lambda) \geqq \eta u & (\text { all } \lambda \geqq(2 n-1) b+\eta ; \text { any } \eta>0), \\
A(\lambda)+B(\lambda) \leqq-\eta u & (\text { all } \lambda \leqq-(2 n-1) b-\eta)
\end{array}
$$

for all $t \geqq t_{0}$.

We think of the set of functions

$$
x_{1}, \cdots, x_{n}
$$

as a solution of (5.1); accordingly

$$
\left|x_{j}\right| \leqq H \quad\left(j=1, \cdots, n ; t \geqq t_{0}\right) .
$$

In consequence of these inequalities of (5.10) and of (5.8a)

$$
\left|p_{i}^{p, k}\right| \leqq b .
$$

Hence by the inequality of Schwarz and by (5.10)

$$
\left|p_{n, i}^{\prime}\right| \leqq e^{-\lambda t} n b u
$$

Accordingly, the function $C(\lambda)$ of (5.12a) will satisfy

$$
|C(\lambda)|^{2} \leqq \sum_{i} z_{i}^{2} \sum_{i}\left(p_{n, i}^{\prime}\right)^{2} \leqq b^{2} e^{-2 \lambda t} n^{3} u^{3}
$$

for all real $\lambda$ and for all $t \geqq t_{0}$. Now, by (5.14), (5.9) and (5.11)

whence

$$
u^{1 / 2} \leqq n^{1 / 2} H e^{\lambda t}
$$

$$
|C(\lambda)| \leqq H n^{2} b u \text {. }
$$

On choosing $\eta$ of (5.13) as

$$
\eta=H n^{2} b+\epsilon \quad(\epsilon>0),
$$

by virtue of (5.12), (5.13) and (5.15) it is concluded that

$$
\frac{1}{2} \frac{d u}{d t} \geqq \epsilon u \quad\left(\text { all } \lambda \geqq b\left(H n^{2}+2 n-1\right)+\epsilon=\lambda_{0}\right) .
$$

Similarly, it is shown that

$$
\frac{1}{2} \frac{d u}{d t} \leqq-\epsilon u \quad\left(\text { all } \lambda \leqq-\lambda_{0}\right)
$$

Therefore

$$
u \geqq c e^{2 \epsilon t} \quad\left(\text { all } \lambda \geqq \lambda_{0}\right), \quad u \leqq c_{1} e^{-2 e t} \quad\left(\text { all } \lambda \leqq-\lambda_{0}\right)
$$

for all $t \geqq t_{0}$; here $c, c_{1}$ are positive constants. Now $\epsilon(>0)$ may be taken arbi- 
trarily small. Thus, on writing $v=x_{1}^{2}+\cdots+x_{n}^{2}$, we find that

$$
\begin{array}{lrl}
\lim _{t} v e^{2 \lambda t}=+\infty & \left(\text { all } \lambda>b\left(H n^{2}+2 n-1\right)\right), \\
\lim _{t} v e^{2 \lambda t}=0 & \left(\text { all } \lambda<-b\left(H n^{2}+2 n-1\right)\right) .
\end{array}
$$

With the aid of reasoning of the type employed subsequent to (3.8), from (5.16) it is inferred that

$$
\left|C\left(x_{1}^{2}+\cdots+x_{n}^{2} \mid e^{t}\right)\right| \leqq 2 b\left(H n^{2}+2 n-1\right),
$$

and

$$
\left|C\left(x_{1}, \cdots, x_{n} \mid e^{t}\right)\right| \leqq b\left(H n^{2}+2 n-1\right) .
$$

In view of $(5.14)$ it is clear that

$$
C\left(x_{1}^{2}+\cdots+x_{n}^{2} \mid e^{t}\right) \geqq 0 .
$$

TheOREM 5.1. Consider the nonlinear differential system, as described in connection with (5.1)-(5.3a). In view of the statement with reference to (5.8)(5.8b) (note transformation (5.5)) there is no loss of generality in assuming (5.8)(5.8b). If a set of functions

$$
x_{1}, \cdots, x_{n}
$$

satisfies the system for all $t \geqq t_{0}$, so that

$$
\left|x_{i}\right| \leqq H \quad(j=1, \cdots, n),
$$

then necessarily

$$
0 \leqq C\left(x_{1}^{2}+\cdots+x_{n}^{2} \mid e^{t}\right) \leqq 2 b\left(H n^{2}+2 n-1\right) .
$$

More precisely, (5.16) will hold.

It is observed that the information supplied by the inequality, last displayed in the above theorem, amounts to an assertion to the effect that $x_{1}^{2}+\cdots+x_{n}^{2}$ cannot approach zero $($ as $t \rightarrow \infty)$ faster than at a certain rate.

Consider the case when, for $t \rightarrow \infty$,

$$
\lim p_{i, j}(t)=\lim p_{i}^{j_{1}, \cdots, i_{n}}(t)=\lim r^{0}=0 .
$$

The function $p_{0}(t)$ of (5.4), (5.4a) will then tend to zero with $1 / t$. There exists a "least" monotone non-increasing function $m(t)$, continuous for $t \geqq t_{0}$, for which

$$
p(t) \leqq m(t), \quad \lim m(t)=0 .
$$


If one had $m\left(t_{1}\right)=0$ for some $t_{1} \geqq t_{0}$ we would have

$$
f_{i}\left(t ; x_{1}, \cdots, x_{n}\right) \equiv 0 \quad\left(\text { all } t \geqq t_{1} ; i=1, \cdots, n\right) ;
$$

the system (5.1) would then be trivial from our point of view. Hence we may take

$$
m(t)>0 .
$$

In (5.16), on letting $v=x_{1}^{2}+\cdots+x_{n}^{2}$, we have

$$
\begin{aligned}
\lim _{t} v \exp \left\{\left[2\left(H n^{2}+2 n-1\right) m(\tau)+\epsilon / 2\right] t\right\} & =+\infty, \\
\lim _{t} v \exp \left\{\left[-2\left(H n^{2}+2 n-1\right) m(\tau)-\epsilon / 2\right] t\right\} & =0
\end{aligned}
$$

for all $\epsilon>0$ and for all $\tau \geqq \tau_{0}$. For any $\epsilon$, such that

$$
0<\epsilon \leqq \epsilon_{0}<4 m\left(t_{0}\right)\left(H n^{2}+2 n-1\right),
$$

the equation

$$
2\left(H n^{2}+2 n-1\right) m(\tau)=\epsilon / 2
$$

will have a solution $\tau=\tau_{\epsilon} \geqq t_{0}$. Substitution of $\tau=\tau_{\epsilon}$ in (5.19) will yield

$$
\lim _{t} v e^{\epsilon t}=\infty, \quad \lim _{t} v e^{-\epsilon t}=0
$$

that is,

$$
C_{s}\left(x_{1}^{2}+\cdots+x_{n}^{2} \mid e^{t}\right)=0 .
$$

We have established

TheOREM 5.2. Consider the nonlinear differential system (5.1). Suppose that the functions, involved, vanish for $t \rightarrow \infty$, as indicated in (5.18). Then for every solution

$$
x_{1}, \cdots, x_{n}
$$

of (5.1), defined for $t \geqq t_{0}$, we shall have (5.20).

With the aid of a reasoning of the type used subsequent to Theorem 3.2 we obtain the following corollary to Theorem 5.2.

Consider the system (5.1). Let $p_{0}(t)$ be the function of (5.4), (5.4a). If the integral

$$
\int_{t_{0}}^{\infty} p_{0}(t) d t
$$

diverges, then for every solution $x_{1}, \cdots, x_{n}$ of (5.1) (with $\left|x_{j}\right| \leqq H$ ) we shall have 


$$
C_{s}\left(x_{1}^{2}+\cdots+x_{n}^{2} \mid \exp \int_{t_{0}}^{t} \rho(t) d t\right)=0
$$

for every continuous function $\rho(t)(\geqq 0)$ such that

$$
\lim _{t \rightarrow \infty} \frac{p_{0}(t)}{\rho(t)}=0 \text {. }
$$

If the integral (5.21) converges (or, more generally, if $\lim p_{0}(t)=0$ ), then for every solution $x_{1}, \cdots, x_{n}$ one has (5.22) for every continuous function $\rho(t)$ $(\geqq 0)$ for which

$$
\lim \frac{p_{0}(t)}{\rho(t)}=0, \quad \int_{t_{0}}^{\infty} \rho(t) d t \text { diverges. }
$$

6. Method of successive approximations. In this section we shall establish certain "growth" properties for solutions of the nonlinear system (5.1), making use of the solutions of the corresponding linear problem

$$
\frac{d x_{j}}{d t}=l_{j}(t ; x)=p_{1, j}(t) x_{1}+\cdots+p_{n, j}(t) x_{n} \quad(j=1, \cdots, n) .
$$

We shall use solutions of (6.1) as the first approximation.

For some continuous function $p^{0}(t)$ we have

$$
\left|p_{j}^{j_{1}, \cdots, j_{n}}(t)\right| \leqq p^{0}(t) .
$$

We shall write

$$
q_{j}=q_{j}(t ; x)=\alpha_{j}\left(t ; x_{1}, \cdots, x_{n}\right)+\beta_{j}\left(t ; x_{1}, \cdots, x_{n}\right),
$$

(6.3a) $\alpha_{j}\left(t ; x_{1}, \cdots, x_{n}\right)=\sum_{j_{1}+\cdots+j_{n}=2} p_{j}^{j_{1}, \cdots, j_{n}}(t) x_{1}^{j_{1}} \cdots x_{n}^{j_{n}}=\sum_{\nu, m=1}^{n} p_{j}^{\nu, m}(t) x_{\nu} x_{m}$,

(6.3b) $\beta_{j}\left(t ; x_{1}, \cdots, x_{n}\right)=\sum_{j_{1}+\cdots+j_{n}=2} r_{j}^{j_{1}, \cdots, j_{n}}(t ; x) x_{1}^{j_{1}} \cdots x_{n}^{j_{n}}=\sum_{\nu, m=1}^{n} r_{j}^{\nu, m}(t ; x) x_{\nu} x_{m}$; here

$$
p_{j}^{\nu, m}(t)=p_{j}^{m, \nu}(t), \quad r_{j}^{\nu, m}(t ; x)=r_{j}^{m, \nu}(t ; x) .
$$

In order to carry out the method of successive approximations a special continuity condition for the functions

$$
r_{j}^{\nu, m}(t ; x)=r_{j}^{\nu, m}\left(t ; x_{1}, \cdots, x_{m}\right)
$$

is needed. It will be convenient to introduce a hypothesis in the nature of a Lipschitz condition. 
HyPOTHEsIs 6.5. The functions (6.4), involved in (6.3b), satisfy inequalities (6.5a) $\left|r_{j}^{\nu, m}\left(t ; z_{1}+x_{1}, \cdots, z_{n}+x_{n}\right)-r_{j}^{\nu, m}\left(t ; z_{1}, \cdots, z_{n}\right)\right| \leqq r_{1}(t) x^{*}\left(t \geqq t_{0}\right)$, whenever

$$
\left|z_{j}\right| \leqq z^{*}, \quad\left|x_{j}\right| \leqq x^{*} \quad(j=1, \cdots, n), \quad z^{*}+x^{*} \leqq H
$$

here $r_{1}(t)$ is continuous.

The following lemma will be stated without proof.

Lemma 6.1. Under Hypothesis 6.5 the functions $q_{j}=q_{j}(t ; x)$, involved in the system (5.1), satisfy the inequalities

$$
\left|q_{j}\left(t ; z_{1}+x_{1}, \cdots, z_{n}+x_{n}\right)-q_{j}\left(t ; z_{1}, \cdots, z_{n}\right)\right| \leqq q_{0}(t)\left(z^{*}+x^{*}\right) x^{*}
$$
$\left(j=1, \cdots, n ; t \geqq t_{0}\right)$, where

$$
q_{0}(t)=2 n^{2}\left(p^{0}(t)+r^{0}(t)\right)+n^{2} H r_{1}(t)
$$

[cf. (6.2), (5.3) $\left.\left(r^{0}=r^{0}(t)\right),(6.5 \mathrm{a})\right]$, whenever (6.5b) holds.

We shall now prove the following result.

LEMMA 6.2. Effecting on $t$, if necessary, a suitable transformation

$$
\left.\tau=\int_{t_{0}}^{t} q^{\prime}(t) d t \quad \text { (continuous } q^{\prime}(t)>0\right),
$$

the function $q_{0}(t)$, involved in (6.6), may be replaced by a constant $q_{0}$. Moreover, it can be arranged at the same time to have the $\left|p_{i, j}(t)\right|$ of (6.1) uniformly bounded.

In fact, if $q_{0}(t)$ is uniformly bounded we may define $q_{0}$ as the least upper bound of $q_{0}(t)$. In the contrary case the integral

$$
\int_{t_{0}}^{\infty} q_{0}(t) d t
$$

will diverge; the transformation (6.7), with $q^{\prime}(t)=q_{0}(t)$, will yield the system

$$
\frac{d x_{j}}{d \tau}=\frac{1}{q_{0}(t)} l_{j}(t ; x)+\frac{1}{q_{0}(t)} q_{j}(t ; x)=\bar{l}_{j}(\tau ; x)+\bar{q}_{j}(\tau ; x),
$$

for which the function $\bar{q}_{0}(\tau)$, corresponding to $q_{0}(t)$, is given by

$$
\bar{q}_{0}(\tau)=1 \quad(0 \leqq \tau<\infty) .
$$

If it were desired to have $q_{0}(t)$ in (6.6) replaced by a constant and at the same time to have the $\left|p_{i, j}(t)\right|$, in (6.1), uniformly bounded, we would proceed as follows. Let $p(t)$ be the "least" continuous function such that

$$
\left|p_{i, j}(t)\right| \leqq p(t) .
$$


If $q_{0}(t)$ is not uniformly bounded, let $q^{\prime}(t)$ be the "least" continuous function such that

$$
q_{0}(t), \quad p(t) \leqq q^{\prime}(t)
$$

Then the integral

$$
\int_{t_{0}}^{\infty} q^{\prime}(t) d t
$$

will be divergent and the transformation (6.7) will produce the desired effect. If $q_{0}(t)$ is uniformly bounded, but $p(t)$ is not, then we still define $q^{\prime}(t)$ as above. The integral (6.8) will be divergent again. The transformation (6.7) will serve the purpose.

The developments in the sequel will be under the following hypothesis relating to the linear problem (6.1).

Hypothesis 6.9. There exists a matrix solution $X(t)=\left(x_{i, j}(t)\right)(i, j=1$, $\cdots, n)$ of the matrix equation

$$
X^{(1)}(t)=X(t) P(t), \quad P(t)=\left(p_{i, j}(t)\right)
$$

such that

$$
\left|x_{i, j}(t)\right| \leqq \phi_{i}(t) \quad\left(i=1, \cdots, n ; t \geqq t_{0}\right),
$$

while the elements $\bar{x}_{i, j}(t)$ from the matrix $X^{-1}(t)=\left(\bar{x}_{i, j}(t)\right)$ satisfy

$$
\left|\bar{x}_{i, j}(t)\right| \leqq \frac{1}{\phi_{j}(t)} \bar{\phi}(t) .
$$

Here the $\phi_{i}(t), \phi(t)$ are continuous. There exists a continuous function $\theta(t)$ so that

$$
\phi_{1}(t) \leqq \phi_{2}(t) \leqq \cdots \leqq \phi_{m}(t) \leqq \cdots \leqq \phi_{n}(t),
$$

$$
\lim \phi_{j}(t)=0
$$
$(j=1, \cdots, m)$,

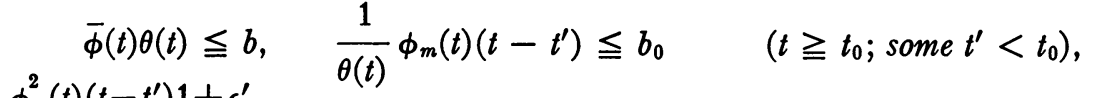
$\frac{\phi_{m}^{2}(t)\left(t-t^{\prime}\right) 1+\epsilon^{\prime}}{\theta(t) \phi_{1}(t)} \rightarrow 0 \quad\left[\right.$ monotonically as $t\left(\geqq t_{0}\right) \rightarrow+\infty$; some $\left.\epsilon^{\prime}>0\right]$,

$$
\lim \frac{\phi_{m}(t)}{\theta(t)}\left(t-t^{\prime}\right)=0 .
$$

Moreover, the functions $\phi_{1}(t) \phi_{\lambda}^{-1}(t)(\lambda=1, \cdots, n)$ are monotone non-increasing.

Further along in the text an important example of linear differential systems will be given for which conditions of Hypothesis 6.9 hold. 
If $C=\left(c_{i, j}\right)$ is a matrix of arbitrary constants with determinant $|C|$, distinct from zero, then

$$
C X(t)=\left(\sum_{\tau} c_{i, \tau} x_{\tau, j}(t)\right)
$$

will constitute a matrix solution of $(6.9 a)$. With $(6.9 \mathrm{~b}),\left(6.9 \mathrm{~d}_{1}\right)$ in view, we form functions

$$
x_{j: 0}(t)=\sum_{\tau=1}^{m} c_{i, \tau} x_{\tau, j}(t) \quad(j=1, \cdots, n) ;
$$

for a fixed $i$ these functions will constitute a solution of (6.9a). Accordingly

$$
x_{j: 0}(t)=c_{1} x_{1, j}(t)+\cdots+c_{m} x_{m, j}(t) \quad(j=1, \cdots, n)
$$

will constitute a solution of the linear system.

A solution of (5.1) will be obtained in the form of series

$$
x_{j}(t)=x_{j: 0}(t)+x_{j: 1}(t)+\cdots+x_{j: v}(t)+\cdots \quad(j=1, \cdots, n),
$$

where

$$
\begin{array}{lr}
\frac{d x_{j: 0}}{d t}=l_{j}\left(t ; x_{: 0}\right) & (j=1, \cdots, n), \\
\frac{d x_{j: \nu}}{d t}=l_{j}\left(t ; x_{: \nu}\right)+g_{j, \nu}(t) & (j=1, \cdots, n ; \nu=1,2, \cdots) ;
\end{array}
$$

here

$$
g_{i, \nu}(t)=q_{j}\left(t ; x_{: \nu-1}+z_{: \nu-2}\right)-q_{j}\left(t ; z_{: \nu-2}\right) \quad(\nu=1,2, \cdots),
$$

where $z:-1=0$ and

$$
z_{j: \nu}=x_{j: 0}+x_{j: 1}+\cdots+x_{j: \nu} \quad(\nu \geqq 0), \quad q_{j}(t ; z:-1)=0 .
$$

The notation above, for instance for $l_{i}\left(t ; x_{: v}\right)$, is to be understood in the sense that

$$
l_{j}\left(t ; x_{: v}\right)=l_{j}\left(t ; x_{1: v}, \cdots, x_{n: v}\right) .
$$

If, with $\nu \geqq 1, g_{j, \nu}(t)$ is thought of as known, the $x_{j: \nu}(j=1, \cdots, n)$ will be given by

$$
x_{j: \nu}(t)=\sum_{\lambda=1}^{n} w^{\lambda, \nu}(t) x_{\lambda, j}(t) \quad(j=1, \cdots, n),
$$

where

$$
w^{\lambda, \nu}(t)=\sum_{\tau=1}^{n} \int^{t} g_{\tau, \nu}(u) \bar{x}_{\tau, \lambda}(u) d u \quad(\lambda=1, \cdots, n)
$$


provided that the integrals here can be evaluated $\left({ }^{8}\right)$. In determinant form the $\bar{x}_{\tau, \lambda}$ are given by

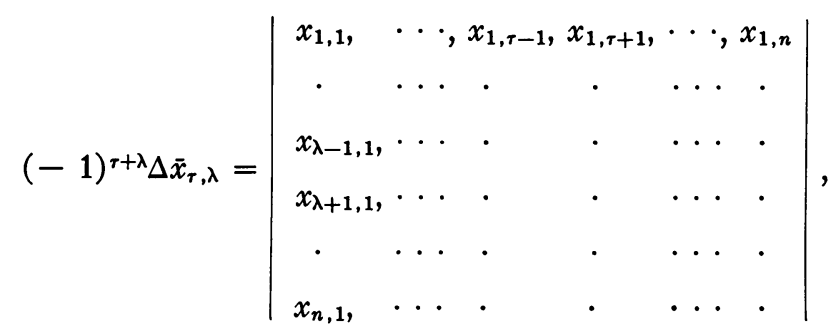

where $\Delta$ denotes the determinant

$$
\Delta=\left|\left(x_{i, j}(t)\right)\right|=\gamma \exp \int^{t}\left(p_{1,1}(u)+\cdots+p_{n, n}(u)\right) d u .
$$

The constants $c_{1}, \cdots, c_{m}$ of (6.10) will be left arbitrary, except for the inequalities

$$
\left|c_{j}\right| \leqq c_{0} \quad\left(j=1, \cdots, m ; c_{0}>0\right) .
$$

We take $c_{0}$ sufficiently small and $t_{0}$ sufficiently great so that

$$
\begin{gathered}
x_{0}^{*}(t)=m c_{0} \phi_{m}(t) \leqq \frac{1}{2} H, \\
q_{0}\left[m c_{0}+2 n n_{0} c_{0}^{2} \theta^{-1}(t) \phi_{m}(t)\left(t-t^{\prime}\right)\right] \leqq 1
\end{gathered}
$$

and so that

$$
\begin{gathered}
x_{1}^{*}(t)=n n_{0} c_{0}^{2} \theta^{-1}(t) \phi_{m}^{2}(t)\left(t-t^{\prime}\right) \leqq 2^{-2} H, \\
\frac{n^{2}}{\epsilon^{\prime}} b b_{0} \leqq \frac{1}{2}
\end{gathered}
$$

In this connection $q_{0}$ is a constant, which according to Lemma 6.2 can be used in (6.6) in place of $q_{0}(t) ;(6.15 \mathrm{~b})$ may be satisfied on noting that, in view of $\left(6.9 \mathrm{~d}_{4}\right)$, the constant $b_{0}$ of $\left(6.9 \mathrm{~d}_{2}\right)$ can be made arbitrarily small by taking $t_{0}$ sufficiently great.

By (6.10), (6.14), (6.9b) and (6.9d)

$$
\left|x_{j: 0}(t)\right| \leqq m c_{0} \phi_{m}(t)=x_{0}^{*}(t)
$$

In consequence of (6.12) and of Lemma 6.1 (with $q_{0}(t)$ replaced by a constant $q_{0}$ )

$$
\left|g_{j, 1}(t)\right|=\left|q_{j}\left(t ; x_{1: 0}, \cdots, x_{n: 0}\right)-q_{j}(t ; 0, \cdots, 0)\right| \leqq q_{0}\left(x_{0}^{*}(t)\right)^{2},
$$

$\left.{ }^{8}\right)$ With the capital letters denoting matrices (of $n^{2}$ elements), it is observed that a matrix solution of $X^{(1)}=X P+G$ may be expressed in the form $X=W X_{0}$, where $X_{0}^{(1)}=X_{0} P$ and $W^{(1)}=G X_{0}^{-1}$. This will yield (6.13), (6.13a). 
where $x_{0}^{*}(t)$ is the function of (6.16). Accordingly, by (6.9c)

$$
\left|g_{\tau, 1}(u) \bar{x}_{\tau, \lambda}(u)\right| \leqq q_{0} m^{2} c_{0}^{2} \phi_{m}^{2}(u) \frac{1}{\phi_{\lambda}(u)} \bar{\phi}(u) \quad\left(u \geqq t_{0}\right) .
$$

Thus, in consequence of $\left(6.9 \mathrm{~d}_{2}\right)$,

$$
\left|g_{\tau, 1}(u) \bar{x}_{\tau, \lambda}(u)\right| \leqq b q_{0} m^{2} c_{0}^{2} \frac{\phi_{m}^{2}(u)}{\theta(u) \phi_{\lambda}(u)} .
$$

Now

$$
I_{\lambda}(u)=\frac{\phi_{m}^{2}(u)\left(u-t^{\prime}\right)^{1+\epsilon^{\prime}}}{\theta(u) \phi_{\lambda}(u)}=\frac{\phi_{m}^{2}(u)\left(u-t^{\prime}\right)^{1+\epsilon^{\prime}}}{\theta(u) \phi_{1}(u)} \cdot \frac{\phi_{1}(u)}{\phi_{\lambda}(u)} .
$$

The two factors in the second member above are monotone non-increasing for $u \geqq t^{\prime}$, as follows from $\left(6.9 \mathrm{~d}_{3}\right)$ and by the statement subsequent to $\left(6.9 \mathrm{~d}_{4}\right)$; the first member in (6.18) will have the same property. Hence

$$
I_{\lambda}(u) \leqq I_{\lambda}(t)
$$

(for all $u \geqq t \geqq t_{0} ;$ cf. $(6.18)$ ).

From (6.17) on using (6.19) we derive

$$
\begin{aligned}
\left|\int_{\infty}^{t} g_{\tau, 1}(u) \bar{x}_{\tau, \lambda}(u) d u\right| & \leqq \int_{t}^{\infty} b q_{0} m^{2} c_{0}^{2} \frac{\phi_{m}^{2}(u)}{\theta(u) \phi_{\lambda}(u)} d u \\
& =b q_{0} m^{2} c_{0}^{2} \int_{t}^{\infty} I_{\lambda}(u)^{\prime}\left(u-t^{\prime}\right)^{-1-\epsilon^{\prime}} d u \\
& \leqq b q_{0} m^{2} c_{0}^{2} I_{\lambda}(t) \int_{t}^{\infty}\left(u-t^{\prime}\right)^{-1-\epsilon^{\prime}} d u \\
& =\frac{1}{\epsilon^{\prime}} b q_{0} m^{2} c_{0}^{2} \frac{\phi_{m}^{2}(t)\left(t-t^{\prime}\right)}{\theta(t) \phi_{\lambda}(t)}
\end{aligned}
$$

In consequence of (6.13a) and (6.20)

$$
\left|w^{\lambda, 1}(t)\right| \leqq \sum_{\tau=1}^{n}\left|\int_{\infty}^{t} g_{\tau, 1}(u) \bar{x}_{\tau, \lambda}(u) d u\right| \leqq n_{0} c_{0}^{2} \frac{\phi_{m}^{2}(t)\left(t-t^{\prime}\right)}{\theta(t) \phi_{\lambda}(t)} ;
$$

here $n_{0}$ has the value assigned in (6.15). Hence, by virtue of (6.13) and (6.9b), one has

$$
\left|x_{j: 1}(t)\right| \leqq \sum_{\lambda=1}^{n}\left|w^{\lambda, 1}(t)\right|\left|x_{\lambda, j}(t)\right| \leqq n n_{0} c_{0}^{2} \theta^{-1}(t) \phi_{m}^{2}(t)\left(t-t^{\prime}\right)=x_{1}^{*}(t) ;
$$

in this connection, we note, $(6.15 a)$ is satisfied.

In view of (6.12) 


$$
g_{j, 2}(t)=q_{j}\left(t ; x_{: 1}+z_{: 0}\right)-q_{j}\left(t ; z_{: 0}\right) \quad\left(z_{: 0}=x_{: 0}\right) .
$$

Thus, by Lemma 6.1,

$$
\begin{aligned}
\left|g_{j, 2}(t)\right| & \leqq q_{0}\left[x_{0}^{*}(t)+x_{1}^{*}(t)\right] x_{1}^{*}(t) \\
& =q_{0}\left[m c_{0}+n n_{0} c_{0}^{2} \frac{\phi_{m}(t)\left(t-t^{\prime}\right)}{\theta(t)}\right] \phi_{m}(t) x_{1}^{*}(t) .
\end{aligned}
$$

Hence, in view of (6.15), $\left|g_{j, 2}(t)\right| \leqq \phi_{m}(t) x_{1}^{*}(t)$.

Whence by $(6.9 \mathrm{c})$ and $\left(6.9 \mathrm{~d}_{2}\right)$

$$
\left|g_{\tau, 2}(u) \bar{x}_{\tau, \lambda}(u)\right| \leqq x_{1}^{*}(u) \frac{\phi_{m}(u)}{\phi_{\lambda}(u)} \bar{\phi}(u) \leqq b x_{1}^{*}(u) \frac{\phi_{m}(u)}{\theta(u) \phi_{\lambda}(u)}
$$

$$
=b n n_{0} c_{0}^{2}\left\{\frac{\phi_{m}(u)\left(u-t^{\prime}\right)}{\theta(u)}\right\}\left\{\frac{\phi_{m}^{2}(u)\left(u-t^{\prime}\right)^{1+\epsilon^{\prime}}}{\theta(u) \phi_{\lambda}(u)}\right\}\left(u-t^{\prime}\right)^{-1-\epsilon^{\prime}}
$$

and, in consequence of $\left(6.9 \mathrm{~d}_{2}\right)$,

$$
\left|g_{\tau, 2}(u) \bar{x}_{\tau, \lambda}(u)\right| \leqq b b_{0} n n_{0} c_{0}^{2} I_{\lambda}(u)\left(u-t^{\prime}\right)^{-1-\epsilon^{\prime}}
$$

for $u \geqq t_{0}$. This, together with (6.19), implies

$$
\left|\int_{\infty}^{t} g_{\tau, 2}(u) \bar{x}_{\tau, \lambda}(u) d u\right| \leqq \frac{1}{\epsilon^{\prime}} b b_{0} n n_{0} c_{0}^{2} I_{\lambda}(t)\left(t-t^{\prime}\right)^{-\epsilon^{\prime}} ;
$$

that is,

$$
\left|\int_{\infty}^{t} g_{\tau, 2}(u) \bar{x}_{\tau, \lambda}(u) d u\right| \leqq \frac{1}{\epsilon^{\prime}} b b_{0} n n_{0} c_{0}^{2} \frac{\phi_{m}^{2}(t)\left(t-t^{\prime}\right)}{\theta(t) \phi_{\lambda}(t)} .
$$

Accordingly by (6.13a)

$$
\left|w^{\lambda, 2}(t)\right| \leqq n n_{2} c_{0}^{2} \frac{\phi_{m}^{2}(t)\left(t-t^{\prime}\right)}{\theta(t) \phi_{\lambda}(t)} \quad\left(n_{2}=\left(1 / \epsilon^{\prime}\right) b b_{0} n n_{0}\right),
$$

and, by virtue of (6.13),

$$
\left|x_{j ; 2}(t)\right| \leqq \sum_{\lambda=1}^{n}\left|w^{\lambda, 2}(t)\right| \phi_{\lambda}(t) \leqq n^{2} n_{2} c_{0}^{2} \frac{\phi_{m}^{2}(t)\left(t-t^{\prime}\right)}{\theta(t)}=x^{*}(2, t) .
$$

In view of the definition of $n_{2}$, given in (6.22), comparison of (6.23) and (6.15a) will yield

$$
x^{*}(2, t)=\frac{n^{2}}{\epsilon^{\prime}} b b_{0} x_{1}^{*}(t) \leqq x_{2}^{*}(t)=2^{-1} x_{1}^{*}(t),
$$

inasmuch as (6.15b) holds. 
Suppose that for some $N>2$ we have

$$
\left|x_{j: \nu}(t)\right| \leqq x_{\nu}^{*}(t)=2^{-(\nu-1)} x_{1}^{*}(t)
$$$$
(\nu=1, \cdots, N-1),
$$

where $x_{1}^{*}(t)$ is from (6.15a). It will be established by induction that (6.24) takes place for $\nu=1,2, \cdots$.

In view of (6.24)

$$
\begin{gathered}
\left|z_{j: N-2}(t)\right| \leqq\left|x_{j: 0}(t)\right|+\cdots+\left|x_{j: N-2}(t)\right| \leqq x_{0}^{*}(t)+x_{1}^{*}(t) \\
+2^{-1} x_{1}^{*}(t)+\cdots+2^{-(N-3)} x_{1}^{*}(t) .
\end{gathered}
$$

For $N=2$ the last member above will consist of $x_{0}^{*}(t)$ only. Thus

$$
\left|z_{j: N-2}(t)\right| \leqq x_{0}^{*}(t)+2 x_{1}^{*}(t)=z^{*}(t)
$$

and, by virtue of $(6.15),(6.15 \mathrm{a})$, one has $z^{*}(t) \leqq H$.

From (6.12) (with $\nu=N$ ) and Lemma 6.1 it is concluded that

$$
\left|g_{j: N}(t)\right|=\left|q_{j}\left(t ; x_{: N-1}+z: N-2\right)-q_{j}\left(t ; z_{: N-2}\right)\right| \leqq q_{0} \cdot\left(z^{*}+x^{*}\right) x^{*}
$$

here $x^{*}, z^{*}$ are from the inequalities

$$
\begin{gathered}
\left|x_{j: N-1}(t)\right| \leqq x^{*}=x_{N-1}^{*}(t)=2^{-(N-2)} x_{1}^{*}(t) \\
\left|z_{j: N-2}(t)\right| \leqq z^{*}=z^{*}(t)
\end{gathered}
$$

Thus, by (6.15) and (6.15b)

$$
\begin{aligned}
\left|g_{i, N}(t)\right| & \leqq q_{0}\left(x_{0}^{*}(t)+2 x_{1}^{*}(t)\right) 2^{-(N-2)} x_{1}^{*}(t) \\
& =q_{0}\left\{m c_{0}+2 n n_{0} c_{0}^{2} \frac{\phi_{m}(t)\left(t-t^{\prime}\right)}{\theta(t)}\right\} \phi_{m}(t) 2^{-(N-2)} x_{1}^{*}(t) \\
& \leqq 2^{-(N-2)} \phi_{m}(t) x_{1}^{*}(t) .
\end{aligned}
$$

In view of (6.26) and (6.9c)

$$
\left|g_{\tau, N}(u) \bar{x}_{\tau, \lambda}(u)\right| \leqq 2^{-(N-2)} x_{1}^{*}(u) \frac{\phi_{m}(u)}{\phi_{\lambda}(u)} \bar{\phi}(u) .
$$

The second member, here, is equal to the second member of (6.21), multiplied by $2^{-(N-2)}$. Carrying out the steps from (6.21) to (6.22), with the indicated modification, from $(6.13 \mathrm{a})$ it is deduced that

$$
\left|\int_{\infty}^{t} g_{\tau, N}(u) \bar{x}_{\tau, \lambda}(u) d u\right| \leqq 2^{-(N-2)} n n_{2} c_{0}^{2} \frac{\phi_{m}^{2}(t)\left(t-t^{\prime}\right)}{\theta(t) \phi_{\lambda}(t)} .
$$

With the aid of (6.13) (for $\nu=N$ ) from (6.27) one finds

$$
\left|x_{j: N}(t)\right| \leqq 2^{-(N-2)} n^{2} n_{2} c_{0}^{2} \frac{\phi_{m}^{2}(t)\left(t-t^{\prime}\right)}{\theta(t)} .
$$


Substituting the expression $n_{2}$ from (6.22) and making use of (6.15b), one obtains

$$
\left|x_{j: N}(t)\right| \leqq 2^{-(N-1)} n n_{0} c_{0}^{2} \frac{\phi_{m}^{2}(t)\left(t-t^{\prime}\right)}{\theta(t)}=2^{-(N-1)} x_{1}^{*}(t)=x_{N}^{*}(t)
$$

for $t \geqq t_{0}$.

Thus, by induction it has been established that (6.24) holds for all $\nu=1,2, \cdots$.

Turning now to the series (6.11) and recapitulating the inequalities

$$
\left|x_{j: 0}(t)\right| \leqq m c_{0} \phi_{m}(t) \leqq 2^{-1} H, \quad\left|x_{j: \nu}(t)\right| \leqq 2^{-(\nu-1)} x_{1}^{*}(t)
$$

where

$$
(\nu=1,2, \cdots)
$$

$$
x_{1}^{*}(t)=\bar{n} c_{0}^{2} \frac{\phi_{m}^{2}(t)\left(t-t^{\prime}\right)}{\theta(t)} \leqq 2^{-2} H \quad\left(\bar{n}=n n_{0}\right)
$$

we obtain

$$
\left|x_{j}(t)-x_{j: 0}(t)\right| \leqq \sum_{\nu=1}^{\infty} 2^{-(\nu-1)} x_{1}^{*}(t)=2 x_{1}^{*}(t) \quad\left(\leqq \frac{1}{2} H\right) .
$$

Theorem 6.1. Consider the nonlinear differential system (5.1) [cf. (6.1), (5.1b), (5.1c), (5.3), (6.2)] under Hypotheses 6.5, 6.9; it is supposed that on making use of Lemma 6.2, the function $q_{0}(t)$ (cf. (6.6a)) involved in (6.6) has been replaced by a constant $q_{0}$. The series (6.11), with integrations suitably performed, will be convergent for $t \geqq t_{0}$ and will represent functions $x_{j}(t)(j=1, \cdots, n)$ constituting a solution of (5.1). This solution is approximated by the solution

$$
x_{j: 0}(t) \quad(j=1, \cdots, n ; \text { cf. }(6.10))
$$

of the linear problem. The approximation is in the sense indicated in (6.29) $[(6.28),(6.28 \mathrm{a})]$. The

$$
x_{j}(t) \quad(j=1, \cdots, n)
$$

contain $m$ arbitrary constants $c_{1}, \cdots, c_{m}$, subject to inequalities

$$
\left|c_{i}\right| \leqq c_{0} \quad\left(i=1, \cdots, m ; c_{0}>0\right) .
$$

The numbers $c_{0}, t_{0}$ are to be chosen according to the italicized statement in connection with (6.15)-(6.15b).

Note. The above developments hold in their essential features when complex values are admitted and $t$ is restricted to a ray. It is also observed that results of the type given in Theorem 6.1 will continue to hold when the hypotheses on the $q_{i}(t ; x)$ (in $(5.1 \mathrm{~b})$ ) are somewhat lightened; in establishing 
Theorem 6.1 we have not used the fact that

$$
\lim r_{j}^{j_{1}, \cdots, j_{n}}(t ; x)=0 \quad\left(\text { as } x_{1}^{2}+\cdots+x_{n}^{2} \rightarrow 0\right) .
$$

The hypotheses originally given, however, are necessary for certain significant further investigations regarding some questions of interest in dynamics.

With a view to an important application of Theorem 6.1 let us consider the following case. After reducing $q_{0}(t)$ in (6.6a) to a constant $q_{0}$, using if necessary a transformation indicated in Lemma 6.2, suppose that the linear problem (6.1) presents the classical "irregular singular point" problem, with the singular point at $t=\infty$; that is, suppose that the $p_{i, j}(t)$ in (6.1) are functions, which at least on the interval $\left(t_{0},+\infty\right)$ are asymptotic $($ at $t=+\infty)$ to series (possibly divergent) of the form

$$
x^{\mu^{\prime} / \kappa}\left[a_{0}+a_{1} x^{-1 / \kappa}+\cdots+a_{\sigma} x^{-\sigma / \kappa}+\cdots\right\rfloor \quad\left(\text { integers } \kappa(>0), \mu^{\prime}\right) .
$$

This, of course, would include constants, polynomials, rational functions, as well as functions analytic in the complex $t$-plane for $|t| \geqq r_{0}>0$ and, possibly, having a pole at $t=\infty$. With regard to the analytic theory of the problem of the "irregular singular point" (for linear differential equations) it is to be said that a complete solution from various points of view has been given by $W . J$. Trjitzinsky $\left({ }^{9}\right)$.

On taking account of the developments given in $(\mathrm{T})$ and of $(6.13 \mathrm{~b}),(6.13 \mathrm{c})$ it may be shown that, except for $\left(6.9 \mathrm{~d}_{1}\right)-\left(6.9 \mathrm{~d}_{4}\right)$, all the conditions of $\mathrm{Hy}$ pothesis 6.9 can be made to hold with the following meaning of the symbols involved. We take $t_{0}(>0)$ sufficiently great; the $\phi_{i}(t)$ are certain functions of the form

$$
\phi_{i}(t)=\left\{\exp \left[\phi_{i, \mu} t^{\mu / p}+\phi_{i, \mu-1} t^{(\mu-1) / p}+\cdots+\phi_{i, 1} t^{1 / p}\right]\right\} t^{r_{i}} \gamma^{\prime}
$$

$\bar{\phi}(t)$ is given by

$$
\begin{array}{r}
{\left[\gamma^{\prime}>0 ; r_{i}, \phi_{i, \nu} \text { real; integers } \mu(>0), p(>0) ;\right.} \\
p=\text { integral multiple of } \kappa] ;
\end{array}
$$

$$
\left.\bar{\phi}(t)=\gamma^{\prime \prime} t^{\alpha} \quad \text { (some } \alpha \geqq 0 ; \gamma^{\prime \prime}>0\right) .
$$

With $t_{0}$ suitably great and the $\phi_{i}(t)$ suitably ordered, not only will conditions $(6.9 \mathrm{~b}),(6.9 \mathrm{c})$ be satisfied for a certain matrix solution $X(t)$ of $(6.9 \mathrm{a})$, but also $(6.9 \mathrm{~d})$ as well as the condition stated subsequent to $\left(6.9 \mathrm{~d}_{4}\right)$ will be satisfied. Now the remaining relations $\left(6.9 \mathrm{~d}_{1}\right)-\left(6.9 \mathrm{~d}_{4}\right)$ will not hold, with

$$
t^{\prime}=0, \quad \theta(t)=t^{-\alpha},
$$

$\left(^{(}\right)$W. J. Trjitzinsky, Analytic theory of linear differential equations, Acta Mathematica, vol. 62 (1934), pp. 167-226, in the sequel referred to as (T).

W. J. Trjitzinsky, Laplace integrals and factorial series in the theory of linear differential and linear difference equations, these Transactions, vol. 37 (1935), pp. 80-146. 
in every case; however, $\left(6.9 \mathrm{~d}_{1}\right)-\left(6.9 \mathrm{~d}_{4}\right)$ will take place in an extensive variety of cases. By (6.30)

$$
\phi_{i}(t)=\gamma^{\prime} t^{r_{i}} \exp \left[t^{\mu / p}\left(\phi_{i, \mu}+t^{-1 / p} \beta_{i}(t)\right)\right],
$$

where the $\left|\beta_{i}(t)\right|$ are uniformly bounded. Having arranged (6.9d), necessarily

$$
\phi_{1, \mu} \leqq \phi_{2, \mu} \leqq \cdots \leqq \phi_{n, \mu},
$$

if we have $\mu>0$. To insure $\left(6.9 \mathrm{~d}_{1}\right)$ it suffices to have

$$
\phi_{1, \mu} \leqq \cdots \leqq \phi_{m, \mu}<0 .
$$

Under $(6.32)$ conditions $\left(6.9 \mathrm{~d}_{1}\right),\left(6.9 \mathrm{~d}_{4}\right)$ will hold. Finally, $\left(6.9 \mathrm{~d}_{3}\right)$ will be secured (with $t_{0}$ sufficiently great) provided that in addition to (6.32) one has

$$
2 \phi_{m, \mu}<\phi_{1, \mu} .
$$

The truth of the latter statement follows from the fact that

$$
t^{2 r_{m}-r_{1}+\alpha+1+\epsilon^{\prime}} \exp \left\{t^{\mu / p}\left[\left(2 \phi_{m, \mu}-\phi_{1, \mu}\right)+t^{1 / p} \beta_{1, m}(t)\right]\right\}
$$

is equal to the first member in $\left(6.9 \mathrm{~d}_{3}\right)$, except for a constant factor, $\left|\beta_{1, m}(t)\right|$ being uniformly bounded.

It is observed that, if (6.32) holds for some $m \geqq 1$, we shall have (6.33) satisfied at least for $m=1$. Accordingly, it is noted that if in (6.30) at least one $\phi_{i, \mu}$, say $\phi_{1, \mu}$, is negative (while $\mu / p>0$ ), all the conditions of Hypothesis 6.9 will be satisfied (with functions (6.30)-(6.31)) on the interval

$$
\left(t_{0},+\infty\right)
$$

( $t_{0}$ suitably great).

With (6.32), (6.33) valid for some $m \geqq 1$, the solution

$$
x_{j}(t) \quad(j=1, \cdots, n)
$$

of the nonlinear problem (5.1), referred to in Theorem 6.1, will be approximated by the solution $x_{j: 0}(t)(j=1, \cdots, n)$ of the linear problem as follows:

$$
\begin{aligned}
\left|x_{j}(t)-x_{j: 0}(t)\right| \leqq & 2 \bar{n} c_{0}^{2} \phi_{m}^{2}(t) t^{1+\alpha} \\
= & \bar{n}_{0} c_{0}^{2} t^{2 r_{m}} \exp \left\{2 t^{\mu / p}\left[\phi_{m, \mu}+t^{-1 / p} \beta_{m}(t)\right]\right\} \\
& \quad\left[t \geqq t_{0} ;\left|\beta_{m}(t)\right| \leqq \beta_{m}<\infty ; \text { constant } \bar{n}_{0}>0 ; \phi_{m, \mu}<0\right] ;
\end{aligned}
$$

here

$$
\left|x_{j: 0}(t)\right| \leqq m c_{0} \phi_{m}(t) \quad(j=1, \cdots, n \text {; cf. }(6.30)) .
$$

If $\mu / p>0$, approximation results of essentially the above type are obtained in all cases for intervals, extending to infinity, along suitable rays

$$
\text { angle of } t=\text { constant, }
$$


provided that on those intervals the nonlinear component of the problem is of the type which we specified above.

We shall have $\mu / p>0$, whenever the singularity, at $t=\infty$, of the linear problem is formally irregular.

UNIVERSITY OF ILLINOIS,

URBANA, ILL.,

The Institute for Advanced Study,

Princeton, N. J. 